\title{
Tunable Pure Spin Supercurrents and the Demonstration of Their Gateability in a Spin-Wave Device
}

\author{
Kun-Rok Jeon $\odot,{ }^{1,2, *}$ Xavier Montiel, ${ }^{3}$ Sachio Komori, ${ }^{1}$ Chiara Ciccarelli, ${ }^{2}$ James Haigh, ${ }^{4}$ Hidekazu Kurebayashi, ${ }^{5}$ \\ Lesley F. Cohen, ${ }^{6}$ Alex K. Chan, ${ }^{6}$ Kilian D. Stenning, ${ }^{6}$ Chang-Min Lee, ${ }^{1}$ \\ Matthias Eschrig, ${ }^{3}$ Mark G. Blamire, ${ }^{1}$ and Jason W. A. Robinson ${ }^{1, \dagger}$ \\ ${ }^{1}$ Department of Materials Science and Metallurgy, University of Cambridge, \\ 27 Charles Babbage Road, Cambridge CB3 OFS, United Kingdom \\ ${ }^{2}$ Cavendish Laboratory, University of Cambridge, Cambridge CB3 OHE, United Kingdom \\ ${ }^{3}$ Department of Physics, Royal Holloway, University of London, \\ Egham Hill, Egham, Surrey TW20 OEX, United Kingdom \\ ${ }^{4}$ Hitachi Cambridge Laboratory, J. J. Thomson Avenue, Cambridge CB3 OHE, United Kingdom \\ ${ }^{5}$ London Centre for Nanotechnology and Department of Electronic and Electrical Engineering at \\ University of College London, London WClH $\mathrm{OlH}$, United Kingdom \\ ${ }^{6}$ Blackett Laboratory, Imperial College London, Prince Consort Road, London SW7 2AZ, United Kingdom
}

(Received 10 January 2020; revised 6 April 2020; accepted 27 May 2020; published 27 July 2020; corrected 6 August 2021)

\begin{abstract}
Recent ferromagnetic resonance experiments and theory of $\mathrm{Pt} / \mathrm{Nb} / \mathrm{Ni}_{8} \mathrm{Fe}_{2}$ proximity-coupled structures strongly suggest that spin-orbit coupling (SOC) in Pt in conjunction with a magnetic exchange field in $\mathrm{Ni}_{8} \mathrm{Fe}_{2}$ are the essential ingredients to generate a pure spin supercurrent channel in Nb. Here, by substituting Pt for a perpendicularly magnetized $\mathrm{Pt} / \mathrm{Co} / \mathrm{Pt}$ spin sink, we are able to demonstrate the role of $\mathrm{SOC}$ and show that pure spin supercurrent pumping efficiency across $\mathrm{Nb}$ is tunable by controlling the magnetization direction of $\mathrm{Co}$. By inserting a $\mathrm{Cu}$ spacer with weak $\mathrm{SOC}$ between $\mathrm{Nb}$ and $\mathrm{Pt} /(\mathrm{Co} / \mathrm{Pt})$ spin sink, we also prove that Rashba-type SOC is key for forming and transmitting pure spin supercurrents across $\mathrm{Nb}$. Finally, by engineering these properties within a single multilayer structure, we demonstrate a prototype superconductor spin-wave device in which lateral spin-wave propagation is gateable via the opening or closing of a vertical pure spin supercurrent channel in $\mathrm{Nb}$.
\end{abstract}

DOI: $10.1103 /$ PhysRevX.10.031020

\section{INTRODUCTION}

Spin-triplet Cooper pairs carry a net spin in addition to charge and are therefore key to the development of superconducting spintronics [1-3], underlying a future revolution in energy-efficient computing. It is established that spin-polarized triplet pairs are generated via spinmixing and spin-rotation processes at magnetically inhomogeneous superconductor-ferromagnet (SC-FM) interfaces [1-3]. Recently, theoretical [4-8] and experimental studies [9-13] have been dedicated to an alternative mechanism for triplet pair creation involving spin-orbit

\footnotetext{
*To whom correspondence should be addressed. jeonkunrok@gmail.com

To whom correspondence should be addressed. jjr33@cam.ac.uk

Published by the American Physical Society under the terms of the Creative Commons Attribution 4.0 International license. Further distribution of this work must maintain attribution to the author(s) and the published article's title, journal citation, and DOI.
}

Subject Areas: Materials Science, Spintronics,
Superconductivity

coupling (SOC) in combination with a magnetic exchange field $h_{\text {ex }}$. In such systems, triplet pair creation depends on the commutation relationship [4-7] between SOC and $h_{\mathrm{ex}}$.

The latter mechanism via SOC in conjunction with $h_{\mathrm{ex}}$ offers a conceptually novel approach to tune superconducting spin currents, as we demonstrate here using ferromagnetic resonance (FMR) spin pumping $[9,14]$. When a perpendicularly magnetized $\mathrm{Pt} / \mathrm{Co} / \mathrm{Pt}$ spin sink is proximity coupled to $\mathrm{Nb}$ (singlet SC) [Fig. 1(a)], the Co thickness $t_{\mathrm{Co}}$-dependent magnetization anisotropy $[15,16]$ changes its effective tilt angle $\theta_{\mathrm{Co}}$ under in-plane (IP) FMR of the IP magnetized $\mathrm{Ni}_{8} \mathrm{Fe}_{2}$ [Fig. 1(b)]. This alters the degree of orthogonality between $h_{\mathrm{ex}}$ and SOC at the interface of $\mathrm{Nb}$ and $\mathrm{Pt} /(\mathrm{Co} / \mathrm{Pt})$ spin sink. Manipulating $\theta_{\mathrm{Co}}$ determines the efficiency with which spin-zero $(S=1$, $\left.s_{z}=0\right)$ triplets [converted from spin singlets $(S=0)$ by the presence of $h_{\mathrm{ex}}$ ] rotate to form equal-spin $\left(S=1, s_{z}= \pm 1\right)$ triplets [4-6]. This enables orthogonality tuning of spinangular-momentum transfer from the precessing $\mathrm{Ni}_{8} \mathrm{Fe}_{2}$ through the proximity-induced equal-spin triplets into singlet $\mathrm{Nb}$ layers, which we call superconducting pure spin currents [9] (see Sec. II of Supplemental Material for 
(a)

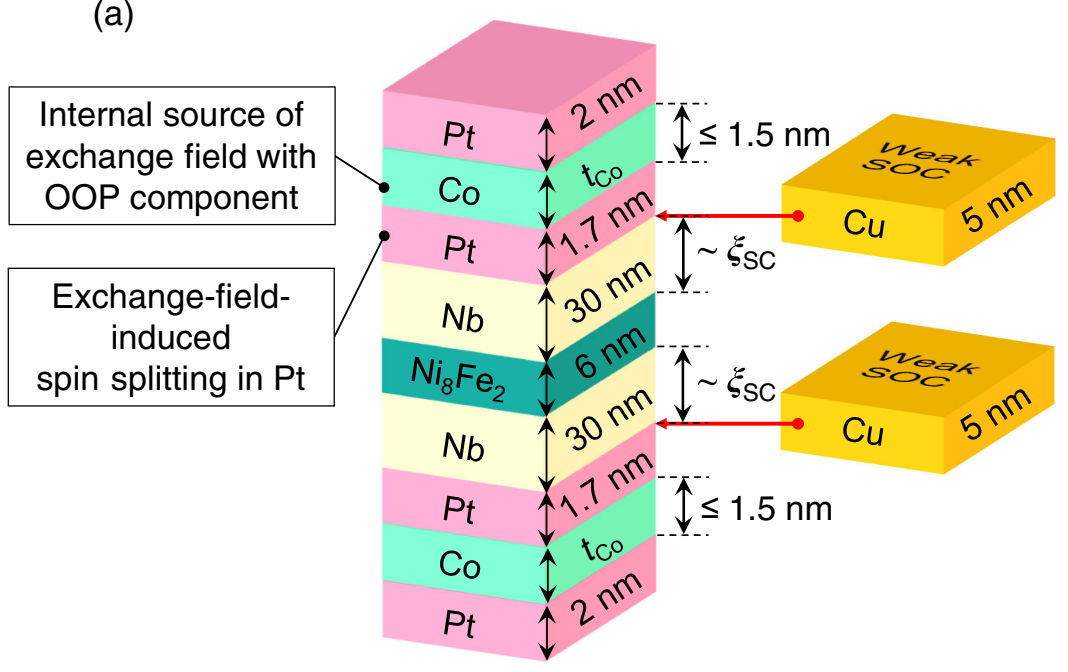

(b)

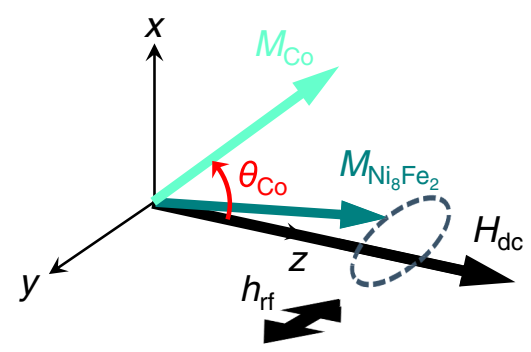

(c)

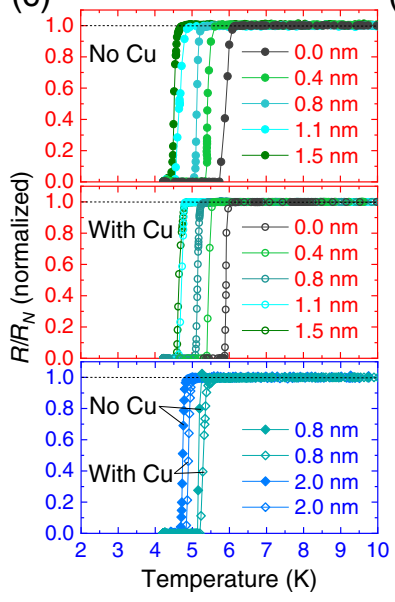

(d)

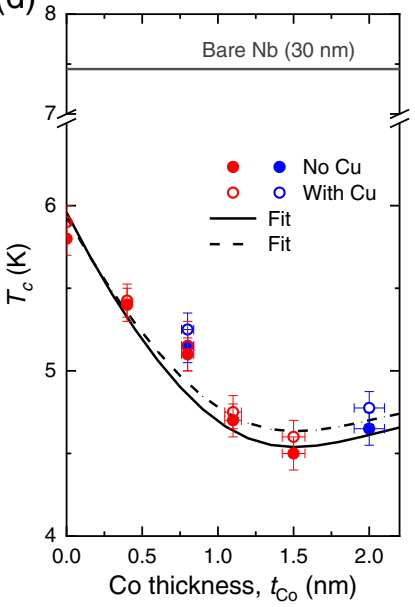

$T=8 \mathrm{~K}, \mathrm{Pt} / \mathrm{Co} / \mathrm{Pt} / \mathrm{Nb}$ only

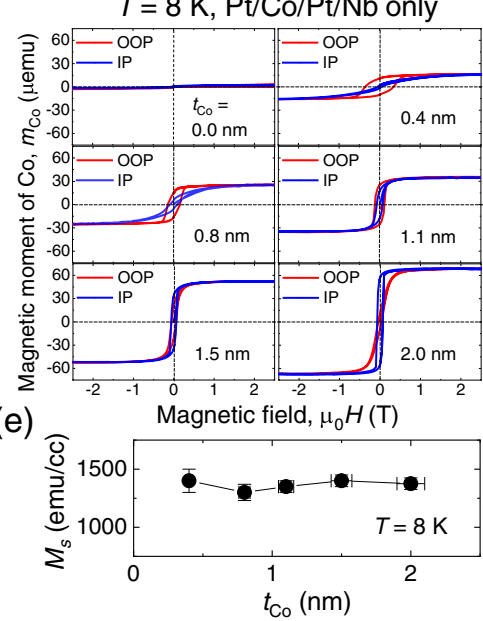

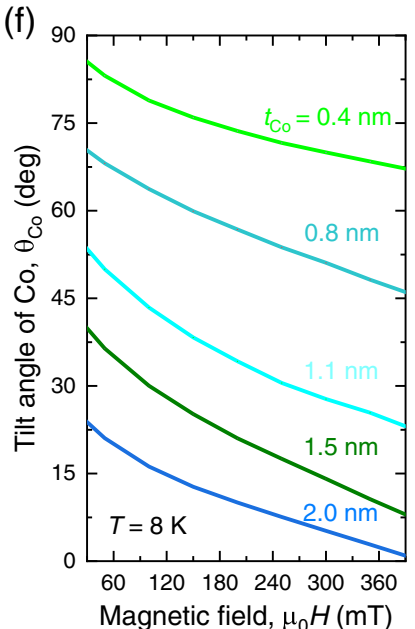

FIG. 1. Principle of the approach and experimental setup (a) Schematic of $\mathrm{Pt}(2.0 \mathrm{~nm}) / \mathrm{Co}\left(t_{\mathrm{Co}}\right) / \mathrm{Pt}(1.7 \mathrm{~nm}) / \mathrm{Nb}(30 \mathrm{~nm}) /$ $\mathrm{Ni}_{8} \mathrm{Fe}_{2}(6 \mathrm{~nm}) / \mathrm{Nb}(30 \mathrm{~nm}) / \mathrm{Pt}(1.7 \mathrm{~nm}) / \mathrm{Co}\left(t_{\mathrm{Co}}\right) / \mathrm{Pt}(2.0 \mathrm{~nm})$ multilayers with different Co thicknesses $t_{\mathrm{Co}}$. The $\mathrm{Cu}$ spacer with weak spin-orbit coupling (SOC) is selected to quench the interfacial Rashba-type $\mathrm{SOC}$ at the interface of $\mathrm{Nb}$ and $\mathrm{Pt} /(\mathrm{Co} / \mathrm{Pt})$ spin sink. (b) Measurement scheme and Cartesian coordinate system used in the present study. (c) Normalized resistance $R / R_{N}$ versus temperature $T$ plots for three different sets of the samples, grown each in a single deposition run. (d) $t_{\mathrm{Co}}$ dependence of the superconducting transition temperature $T_{c}$ of the sample sets with and without $\mathrm{Cu}(5 \mathrm{~nm})$ spacer layers; for comparison, $T_{c}$ of a bare $\mathrm{Nb}(30 \mathrm{~nm})$ film is also shown. The black solid (dashed) line is a fit to estimate the effective values of coherence length and interface transparency (see the Appendix) for the $\mathrm{Cu}$-absent (Cu-present) samples. (e) In-plane and out-of-plane magnetization hysteresis $m(H)$ curves of $\mathrm{Pt}(2.0 \mathrm{~nm}) / \mathrm{Co}\left(t_{\mathrm{Co}}\right) / \mathrm{Pt}(1.7 \mathrm{~nm}) / \mathrm{Nb}(30 \mathrm{~nm})$-only films, measured at $8 \mathrm{~K}$. The diamagnetic background signal from the sample hold is subtracted. The bottom panel summarizes the $t_{\mathrm{Co}}$ dependence of the saturation magnetization $M_{s}$. (f) Effective tilt angle $\theta_{\mathrm{Co}}$ of the Co layer estimated from (e) using the Stoner-Wohlfarth model, in which only the corresponding regime of the in-plane $m(H)$ curves to the ferromagnetic resonance measurement condition and sequence (i.e., from high to low field; see the Appendix) is considered.

the calculated spatial dependence of the equal-spin triplets [17]). Such transmitted spin currents to $\mathrm{Pt} / \mathrm{Co} / \mathrm{Pt}$ spin sinks result in the enhanced spin pumping or transfer which is then probed by measuring the FMR linewidth broadening (Gilbert damping increase) of the middle $\mathrm{Ni}_{8} \mathrm{Fe}_{2}$ layer $[9,14]$.

To demonstrate our approach, we perform a series of FMR measurements on $\mathrm{Pt} / \mathrm{Co} / \mathrm{Pt} / \mathrm{Nb} / \mathrm{Ni}_{8} \mathrm{Fe}_{2} / \mathrm{Nb} / \mathrm{Pt} / \mathrm{Co} / \mathrm{Pt}$ multilayers [Fig. 1(a)]. The ultrathin $(\leq 1.5 \mathrm{~nm})$ perpendicularly magnetized Co layers serve as an internal source of $h_{\mathrm{ex}}$ to the neighboring (inner) Pt layers, supplying spontaneous spin splitting $[18,19]$ with out-of-plane (OOP) polarization [Fig. 1(a)]. The outer Pt layers boost the perpendicular anisotropy of the Co as well as the total effective spin conductance of $\mathrm{Pt} / \mathrm{Co} / \mathrm{Pt}$ trilayers [20] while suppressing the emergence of a noncollinear magnetic ground state 
(e.g., magnetic Skrymion) owing to the structural symmetry and cancellation of top and bottom Dzyaloshinskii-Moriya interactions (DMIs) [21]. A weak DMI and thereby the absence of magnetic Skyrmions in our $\mathrm{Pt} / \mathrm{Co} / \mathrm{Pt}$ symmetric structures are confirmed by magnetic force microscopy (MFM) (see the Appendix), which is in good agreement with previous experimental reports [22,23]. By inserting a thin $\mathrm{Cu}$ spacer with weak SOC at the interface between $\mathrm{Nb}$ and $\mathrm{Pt} /(\mathrm{Co} / \mathrm{Pt})$ layers [Fig. 1(a)], we are able to separate the contribution of interfacial Rashba-type SOC at the $\mathrm{Nb} / \mathrm{Pt}$ to the $\theta_{\mathrm{Co}}$-dependent superconducting spin-pumping efficiency from other contributions such as stray fields and to compare it with the prediction from spin-triplet proximity theory $[4-6,8]$.

\section{RESULTS AND DISCUSSION}

We first measure the $t_{\mathrm{Co}}$ dependence of the superconducting transition $T_{c}$ [Fig. 1(c)] for a series of multilayers with and without $\mathrm{Cu}$ spacers. $T_{c}$ decreases rapidly with increasing $t_{\mathrm{Co}}$ until it reaches about $1.5 \mathrm{~nm}$, where it slightly increases. No significant change in $T_{c}\left(t_{\mathrm{Co}}\right)$ appears with the addition of the $\mathrm{Cu}$ spacer, consistent with its long (thermal) coherence length of several hundred nanometers [3]. In analogy with the original consideration on the nonuniform superconducting state [24,25], such nonmonotonic $T_{c}$ behavior has been discussed based on a spatial modulation of the superconducting order parameter due to Cooper pairs acquiring a nonzero net momentum in the presence of $h_{\mathrm{ex}}$, in particular, for SC-FM multilayers or SC-FM bilayers with FM thickness $t_{\mathrm{FM}}$ of the order of the coherence length $\xi_{\mathrm{FM}}$, which leads to a damped oscillatory behavior of the order parameter [26]. A quantitative analysis (see the Appendix) of the $T_{c}$ data [black lines in Fig. 1(d)] gives an effective $\xi_{\mathrm{FM}}$ of 1.4-1.6 nm and interface transparency $\gamma_{B}=0.18-0.20$ for our samples, which are in reasonable agreement with those obtained from $\mathrm{Nb} / \mathrm{FM}$ [27] bilayers and $\mathrm{Nb} / \mathrm{Cu} / \mathrm{FM}$ trilayers [28] with strong FMs.

The $t_{\mathrm{Co}}$-dependent magnetization anisotropy of the $\mathrm{Pt} / \mathrm{Co} / \mathrm{Pt}$ spin sinks can be independently characterized by static magnetometry measurements on $\mathrm{Pt} / \mathrm{Co} / \mathrm{Pt} / \mathrm{Nb}-$ only films with different $t_{\mathrm{Co}}$. Figure 1(e) shows the typical magnetization hysteresis $m(H)$ curves obtained at $8 \mathrm{~K}$ by applying the external magnetic field $\mu_{0} H$ parallel and perpendicular to the film plane. At low $t_{\mathrm{Co}}(\leq 0.8 \mathrm{~nm})$, the easy axis of the Co magnetization $M_{\mathrm{Co}}$ is OOP, indicating that the ultrathin Co sandwiched between two Pt layers has well-established perpendicular magnetization anisotropy (PMA), as expected for the $\mathrm{Pt} 5 d-$ Co $3 d$ orbital hybridization at either $\mathrm{Pt} / \mathrm{Co}$ interface plus SOC [15]. As $t_{\mathrm{Co}}$ approaches $1.5 \mathrm{~nm}$, the predominant magnetization anisotropy changes from OOP to IP, exhibiting the reorientation transition [16]. Using the relationship [16] $\mu_{0} H_{\text {ani }} M_{s} / 2=K_{\text {eff }}$, where $\mu_{0} H_{\text {ani }}$ is the anisotropy field and $M_{s}$ is the saturation magnetization, the effective PMA energy $K_{\text {eff }}$ is estimated for $t_{\text {Co }} \leq 0.8 \mathrm{~nm}$ to be $\sim 1 \mathrm{MJ} \mathrm{m}^{-3}$, comparable to typical values of the perpendicularly magnetized $\mathrm{Pt} / \mathrm{Co} / \mathrm{Pt}$ trilayers [29].

Assuming coherent rotation of $M_{\mathrm{Co}}$ from OOP under the application of IP resonance fields $\mu_{0} H_{\text {res }}$ for the middle $\mathrm{Ni}_{8} \mathrm{Fe}_{2}$, the effective $\theta_{\mathrm{Co}}$ can be estimated using the simple Stoner-Wohlfarth model where $\theta_{\mathrm{Co}}=\arccos \left[M\left(\mu_{0} H_{\text {res }}\right) /\right.$ $\left.M_{s}\right]$. We then achieve discrete tilt states of the $\mathrm{Pt} / \mathrm{Co} / \mathrm{Pt}$ spin sinks from OOP to IP [Fig. 1(f)], which are systematically controllable by varying $t_{\mathrm{Co}}$. Note that from a MFM study (Fig. 5), the typical dimension of Co magnetic domains (a few microns) in our structure is found to be approximately 2 orders of magnitude larger than both the superconducting coherence length of $\mathrm{Nb}$ thin film $(\leq 40 \mathrm{~nm})$ and the domain wall width $\Delta_{\mathrm{DW}}$ of the perpendicularly magnetized Co layer $(8.6 \mathrm{~nm}$ at $300 \mathrm{~K})$ [30]. In addition, given that $\Delta_{\mathrm{DW}}$ is inversely proportional to $K_{\text {eff }}$ and $M_{s}$ [30,31], $\Delta_{\text {DW }}$ is expected to narrow even further at a lower $T$. This rules out any possible contribution of domain walls and associated magnetic inhomogeneities to the superconducting-state FMR damping enhancement. One can thus assume that within the coherence length which determines the active regime of the triplet proximity effect, the Co magnetization is homogeneous and it rotates coherently under the application of an IP $\mu_{0} H_{\text {res. }}$.

We next show the influence of the tilt states on the superconducting spin-pumping efficiency, namely that the associated orthogonality between $h_{\mathrm{ex}}$ and SOC at the $\mathrm{Nb} / \mathrm{Pt} /(\mathrm{Co} / \mathrm{Pt})$ interface strongly modifies the spinangular-momentum transfer in the superconducting state. Figures 2(a) and 2(b) show the microwave frequency $f$ dependence of FMR data for the $\mathrm{Cu}$-absent (Cu-present) samples, taken above and below $T_{c}$ of the $\mathrm{Nb}$ layers. From this, we extract the effective Gilbert damping $\alpha$, which provides a measure $[9,14,19]$ of the net spin current flow out of the precessing $\mathrm{Ni}_{8} \mathrm{Fe}_{2}$, and the effective saturation magnetization $\mu_{0} M_{s}$ (see the Appendix).

The extracted $\alpha$ and $\mu_{0} M_{s}$ values are plotted as a function of $t_{\mathrm{Co}}$ in Fig. 2(c). In the normal state $\left(T / T_{c}>1\right), \alpha$ is almost $t_{\mathrm{Co}}$ independent for both sample sets and there is a small decrease in the magnitude by introducing the $\mathrm{Cu}$ spacers. This means that the presence of ultrathin $\mathrm{Co}$ $(\leq 2 \mathrm{~nm})$ and $\mathrm{Cu}(5 \mathrm{~nm})$ layers hardly changes the normalstate spin-pumping behavior, as expected from their small spin conductances [20] relative to Pt, and the three layers $(\mathrm{Co}, \mathrm{Cu}, \mathrm{Pt})$ are all approximately spin transparent [33] with each other due to their similar crystal and electronic structures.

In the superconducting state $\left(T / T_{c}<1\right)$, a significant $t_{\mathrm{Co}}$-dependent enhancement of $\alpha$ appears and is strongly affected by the addition of $\mathrm{Cu}$. For the $\mathrm{Cu}$-absent multilayers, as $t_{\mathrm{Co}}$ increases, the superconducting-state damping enhancement (indicating the enhanced spin flow or transfer mediated most likely by equal-spin triplet pairing) $[8,14,19]$ rapidly rises until reaching $0.8 \mathrm{~nm}$ and then slowly decreases for thicker Co layers, resulting in a 
(a)

$\mathrm{No} \mathrm{Cu}$

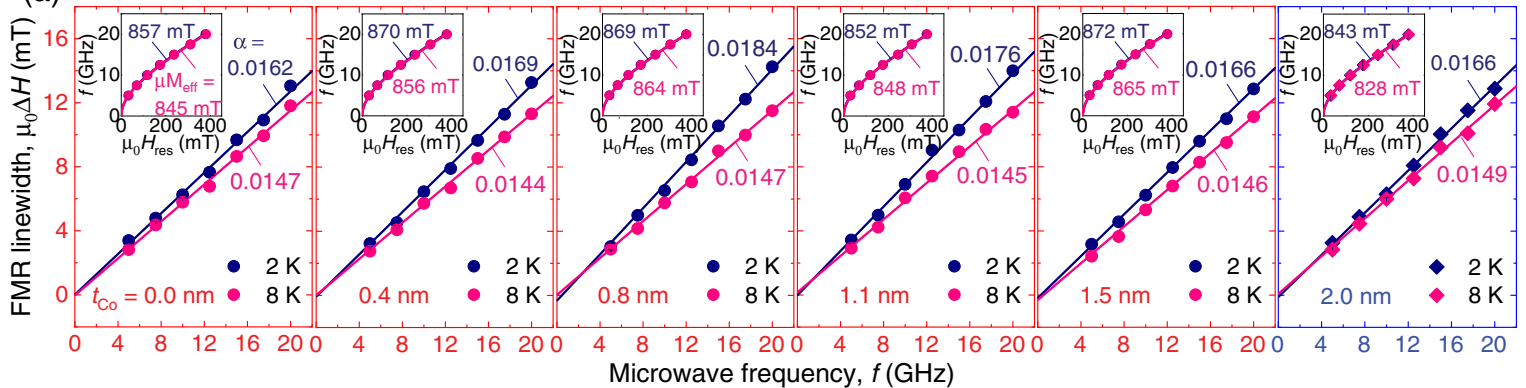

With $\mathrm{Cu}$

(b)
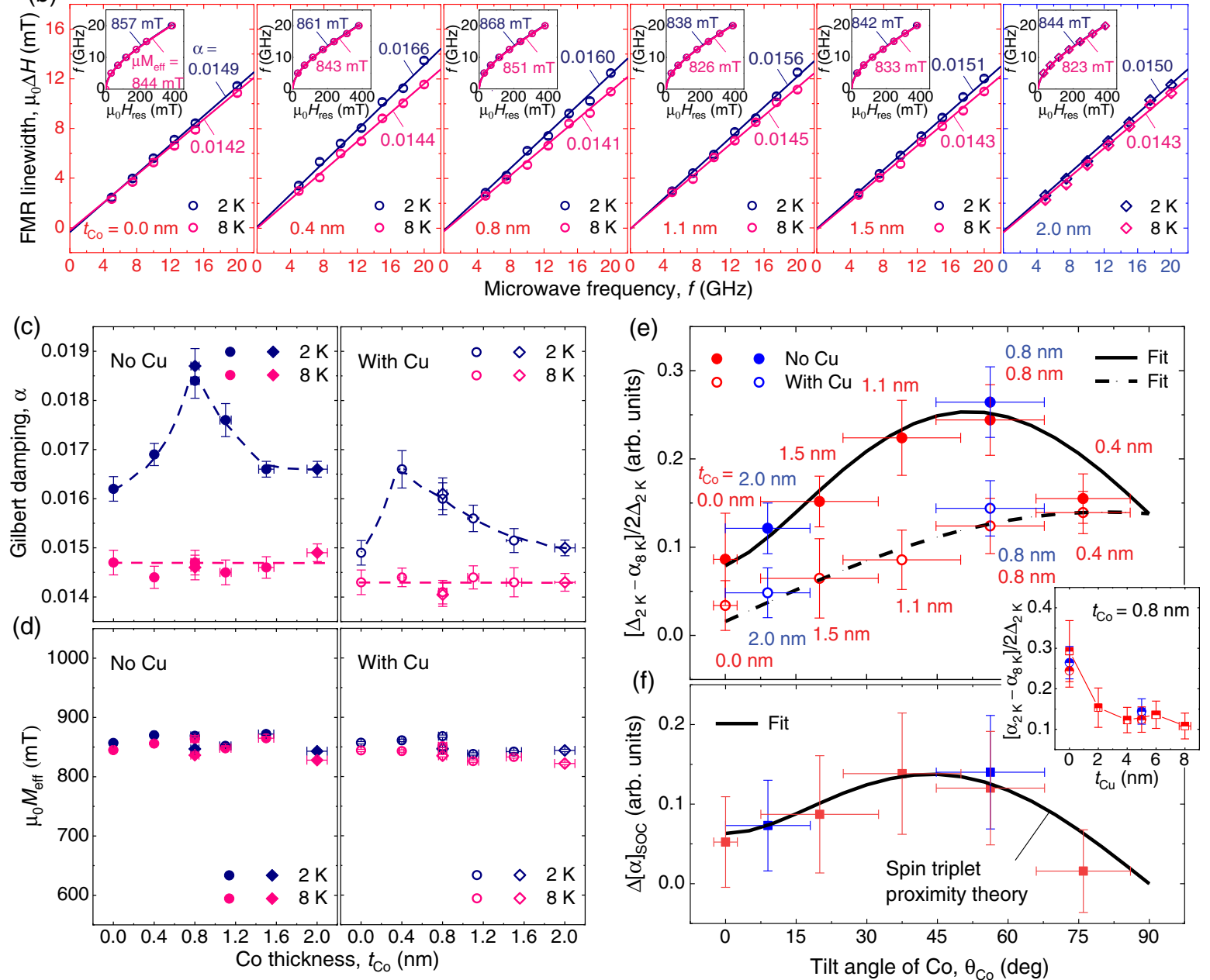

FIG. 2. Correlation of Co tilt angle with superconducting spin-pumping efficiency. (a) Microwave frequency $f$ dependence of ferromagnetic resonance (FMR) absorption for symmetric $\mathrm{Pt}(2.0 \mathrm{~nm}) / \mathrm{Co}\left(t_{\mathrm{Co}}\right) / \mathrm{Pt}(1.7 \mathrm{~nm}) / \mathrm{Nb}(30 \mathrm{~nm}) / \mathrm{Ni}_{8} \mathrm{Fe}_{2}(6 \mathrm{~nm}) / \mathrm{Nb}(30 \mathrm{~nm}) /$ $\mathrm{Pt}(1.7 \mathrm{~nm}) / \mathrm{Co}\left(t_{\mathrm{Co}}\right) / \mathrm{Pt}(2.0 \mathrm{~nm})$ samples with various Co thicknesses, taken above and below $T_{c}$ of the couple Nb. From this, one can extract the (effective) Gilbert(-type) damping $\alpha$ and the (effective) saturation magnetization $\mu_{0} M_{s}$. (b) Data equivalent to (a) but for symmetric $\mathrm{Pt}(2.0 \mathrm{~nm}) / \mathrm{Co}\left(t_{\mathrm{Co}}\right) / \mathrm{Pt}(1.7 \mathrm{~nm}) / \mathrm{Cu}(5 \mathrm{~nm}) / \mathrm{Nb}(30 \mathrm{~nm}) / \mathrm{Ni}_{8} \mathrm{Fe}_{2}(6 \mathrm{~nm}) / \mathrm{Nb}(30 \mathrm{~nm}) / \mathrm{Cu}(5 \mathrm{~nm}) / \mathrm{Pt}(1.7 \mathrm{~nm}) / \mathrm{Co}\left(t_{\mathrm{Co}}\right) / \mathrm{Pt}(2.0 \mathrm{~nm}) \operatorname{samples}$. Note that in any case, the zero-frequency line broadening $\mu_{0} \Delta H_{0}$ due to long-range magnetic inhomogeneities is less than $|0.5 \mathrm{mT}|$ and the FMR linewidth $\mu_{0} \Delta H$ scales linearly with $f$, indicating the high quality of the samples and the absence of two-magnon scattering [32]. Extracted $\alpha(\mathrm{c})$ and $\mu_{0} M_{s}(\mathrm{~d})$ values as a function of $t_{\mathrm{Co}}$ for the samples with and without the $\mathrm{Cu}$ spacer. The dashed lines are guide to the eyes. (e) Damping difference across $T_{c}$, denoted as $\left[\alpha_{2 K}-\alpha_{8 K}\right] / 2 \Delta_{2 K}$, where $2 \Delta$ is the superconducting gap at $2 \mathrm{~K}$ calculated from the measured $T_{c}$ [Fig. 1(d)], as a function of the (effective) Co tilt angle $\theta_{\mathrm{Co}}$. The black solid (dashed) line is a fit from spin-triplet proximity theory [4-6,8] for the $\mathrm{Cu}$-absent (Cu-present) samples (Secs. 1 and 2 of Supplemental Material [17]). (f), Interfacial SOC contribution $\Delta[\alpha]_{\text {SOC }}$, separated by taking the difference between the $\left[\alpha_{2 K}-\alpha_{8 K}\right] / 2 \Delta_{2 K}$ data (e) with and without the $\mathrm{Cu}$ spacer. The black solid line is a theoretical fit based on Rashba-type SOC-induced triplet paring [6,8] (Secs. 1 and 2 of Supplemental Material [17]). Here, the amplitude and component of Rashba SO field and the exchange field strength are only adjustable parameters to get to the theoretical fit. The inset of (e) and (f) shows $\left[\alpha_{2 K}-\alpha_{8 K}\right] / 2 \Delta_{2 K}$ data as a function of Cu spacer thickness $t_{\mathrm{Cu}}$ for the $t_{\mathrm{Co}}=0.8 \mathrm{~nm}$ samples (Supplemental Material, Sec. IV [17]). The red and blue symbols in (c) and (d) represent independent sets of the samples grown each in a single deposition run. 
maximum at $t_{\mathrm{Co}} \approx 0.8 \mathrm{~nm}$. For the $\mathrm{Cu}$-present samples, the overall amplitude of damping enhancement diminishes compared with the $\mathrm{Cu}$-absent samples and the maximum moves to a lower value of $t_{\mathrm{Co}}(0.4 \mathrm{~nm})$. Since this nontrivial enhancement of $\alpha\left(t_{\mathrm{Co}}\right)$ occurs in the ultrathin regime $\left(t_{\mathrm{Co}} \leq 2 \mathrm{~nm}\right.$, about one order of magnitude smaller than the spin diffusion length [34]) only for the superconducting state, it must reflect how the tilt states of the $\mathrm{Pt} / \mathrm{Co} / \mathrm{Pt}$ spin sinks correlate with the superconducting spin transport.

To elucidate this, we have plotted the damping difference across $T_{c}$, defined as $\left[\alpha_{2 K}-\alpha_{8 K}\right] / 2 \Delta_{2 K}$ where $2 \Delta$ is the superconducting gap at $2 \mathrm{~K}$ calculated from the measured $T_{c}$ [Fig. 1(d)], with and without the $\mathrm{Cu}$ versus the effective $\theta_{\mathrm{Co}}$ [Fig. 2(e)]. In the absence of the $\mathrm{Cu},\left[\alpha_{2 K}-\alpha_{8 K}\right] / 2 \Delta_{2 K}$ rapidly rises with increasing $\theta_{\mathrm{Co}}$ from $0^{\circ}$ to $56^{\circ}$ followed a fall for a higher angle. However, this characteristic angular dependence vanishes when the $\mathrm{Cu}$ spacer (with weak SOC) is present: the damping difference increases monotonically and slowly up to the highest angle and saturates to a value similar to the $\mathrm{Cu}$-absent $\theta_{\mathrm{Co}} \approx 76^{\circ}\left(t_{\mathrm{Co}}=0.4 \mathrm{~nm}\right)$ sample.

There are, in principle, two different sources of proximityinduced triplet pairing which can contribute to the characteristic angular dependence observed in our experimental setup. First, it is well known that magnetization noncollinearity (or inhomogeneity) [1-3,35] between two FMs separated by a SC with a thickness of the order of the coherence length can generate equal-spin triplets through the entire structure. The equal-spin triplet density is then ascribed to the relative magnetization angle $\theta$ between the two FMs [35]: $\propto \mathbf{M}_{\mathrm{Co}} \times \mathbf{M}_{\mathrm{Py}} \propto \sin (\theta)\left(\mathrm{Py}\right.$ is $\left.\mathrm{Ni}_{8} \mathrm{Fe}_{2}\right)$. This explains why our $\theta_{\mathrm{Co}} \approx 76^{\circ}\left(t_{\mathrm{Co}}=0.4 \mathrm{~nm}\right)$ samples show larger enhancements than the $\theta_{\mathrm{Co}} \approx 9^{\circ}\left(t_{\mathrm{Co}}=2.0 \mathrm{~nm}\right)$ samples [Fig. 2(e)]. Second, even for a single magnetically homogeneous FM, the equal-spin triplet correlation is generated by introducing a strongly SO coupled interface (e.g., Pt) between the FM and SC [4-6,8]. In this case, the singlet-triplet conversion efficiency is predicted to scale with the degree of orthogonality between SOC and $h_{\mathrm{ex}}$, or equivalently, the cross product of the SO vector operator $\left[\hat{A}_{k},\left[\hat{A}_{k}, h^{a} \sigma^{a}\right]\right]$ and the exchange field operator $h^{a} \sigma^{a}$. Here $\hat{A}_{k=x, y, z}$ is the vector potential describing the form of the SOC, for instance, the Rashba constant $\alpha_{R}$ (Dresselhaus constant $\beta_{D}$ ) due to the interface (bulk) inversion asymmetry. $\sigma^{a}\left(h^{a}\right)$ with $a=x, y, z$ is the vector of Pauli matrices (exchange field).

For a metallic vertical structure with atomically flat interfaces, the vector potential can be approximated as $\hat{A}_{x} \approx 0, \hat{A}_{y} \approx-\beta_{D} \sigma^{y}+\alpha_{R} \sigma^{z}, \hat{A}_{z} \approx \beta_{D} \sigma^{z}-\alpha_{R} \sigma^{y}[4,5]$. With finite Rashba $\left(\alpha_{R} \neq 0\right)$ and zero Dresselhaus $\left(\beta_{D}=0\right)$ contributions to the SOC $[6,8]$, as relevant to our experimental setup, a sinusoidal maximum of the equal-spin triplet correlation is expected when the canting angle between IP and OOP components of $h_{\mathrm{ex}}$ becomes $45^{\circ}$. In such a case, the overall triplet density is quadratic in $\alpha_{R}$ and very sensitive to details of the spin-orbit coupled interface. The addition of a thin $\mathrm{Cu}$ spacer layer [36] at the spin-orbit coupled interface is sufficient to quench the interfacial Rashba-type SOC and provide the key test experiment for the mechanism responsible here [see inset of Figs. 2(e) and 2(f)].

We emphasize that for the $t_{\mathrm{Co}}=0.8 \mathrm{~nm}$ sample set with various $\mathrm{Cu}$ spacer thicknesses $t_{\mathrm{Cu}}$ prepared in $a$ single deposition run [red symbols in the inset of Figs. 2(e) and 2(f)], FMR damping of the middle $\mathrm{Ni}_{8} \mathrm{Fe}_{2}$ layer is $t_{\mathrm{Cu}}$ independent in the normal state (see Fig. S4 in Supplemental Material [17]). This proves that the addition of $\mathrm{Cu}$ has no measurable effect on the normal-state FMR. However, there is a dramatic decrease by a factor of 2 in the superconducting spin-pumping efficiency with increasing $t_{\mathrm{Cu}}$, meaning that the presence of the $\mathrm{Cu}$ spacer strongly modifies the superconducting-state FMR response due to the quenching of the Rashba SOC at the interface between $\mathrm{Nb}$ and $\mathrm{Pt} /(\mathrm{Co} / \mathrm{Pt})$ layers. Furthermore, the differences in the superconducting FMR response between sample sets with versus without a $\mathrm{Cu}$ spacer cannot be justified on the basis of slight variations in the Co static magnetization as such variations would also affect the normal-state FMR.

We note that nonvanishing of $\hat{A}_{x}(\neq 0)$, as would be expected from nonideal interfaces where the OOP component of the Rashba SO field with respect to the local interface plane survives on a scale of the coherence length [7], allows the equal-spin triplet to be generated locally even with a purely IP magnetized FM $\left(h^{x}=0\right)$. Each triplet channel is then able to transport spin angular momentum from the precessing $\mathrm{FM}\left(\mathrm{Ni}_{8} \mathrm{Fe}_{2}\right)$ through a singlet $\mathrm{SC}(\mathrm{Nb})$ to a spin dissipative bath (Pt spin sink) independently even if the spatial average of net polarization of total triplet channels over the entire interface plane becomes zero. This is a likely mechanism for our previous FMR experiments $[9,19]$ and for the $t_{\mathrm{Co}}=0$ samples [Fig. 2(e)]. When the Pt spin sink is substituted for the perpendicularly magnetized $\mathrm{Pt} / \mathrm{Co} / \mathrm{Pt}$ spin sink, a global triplet channel opens in addition to the local channels, maximizing the overall superconducting spin-pumping efficiency at $\theta_{\mathrm{Co}} \approx 45^{\circ}$ [Fig. 2(e)].

By taking all these effects together, we can arrive at good fits to $\left[\alpha_{2 K}-\alpha_{8 K}\right] / 2 \Delta_{2 K}$ versus $\theta_{\text {Co }}$ data for both sample sets [black solid and dashed lines in Fig. 2(e); see Secs. 1 and 2 of Supplemental Material [17] ], thereby reasonably reproducing the experimental results and capturing the underlying physics. To focus on the second SOC mechanism, in particular for the interfacial contribution, we take the difference between the data with and without the $\mathrm{Cu}$ spacer [Fig. 2(f)]. We then find an approximately sinusoidal maximum at $\theta_{\mathrm{Co}} \approx 45^{\circ}$, which is in good agreement with the Rashba SOC-induced triplet pairing $[6,8]$ described above. The data described above provide a proof-ofconcept result demonstrating the orthogonality tuning of superconducting spin currents.

To understand better the FMR absorption data of symmetric structures [Fig. 2], we also measure the $t_{\text {Co }}$ 

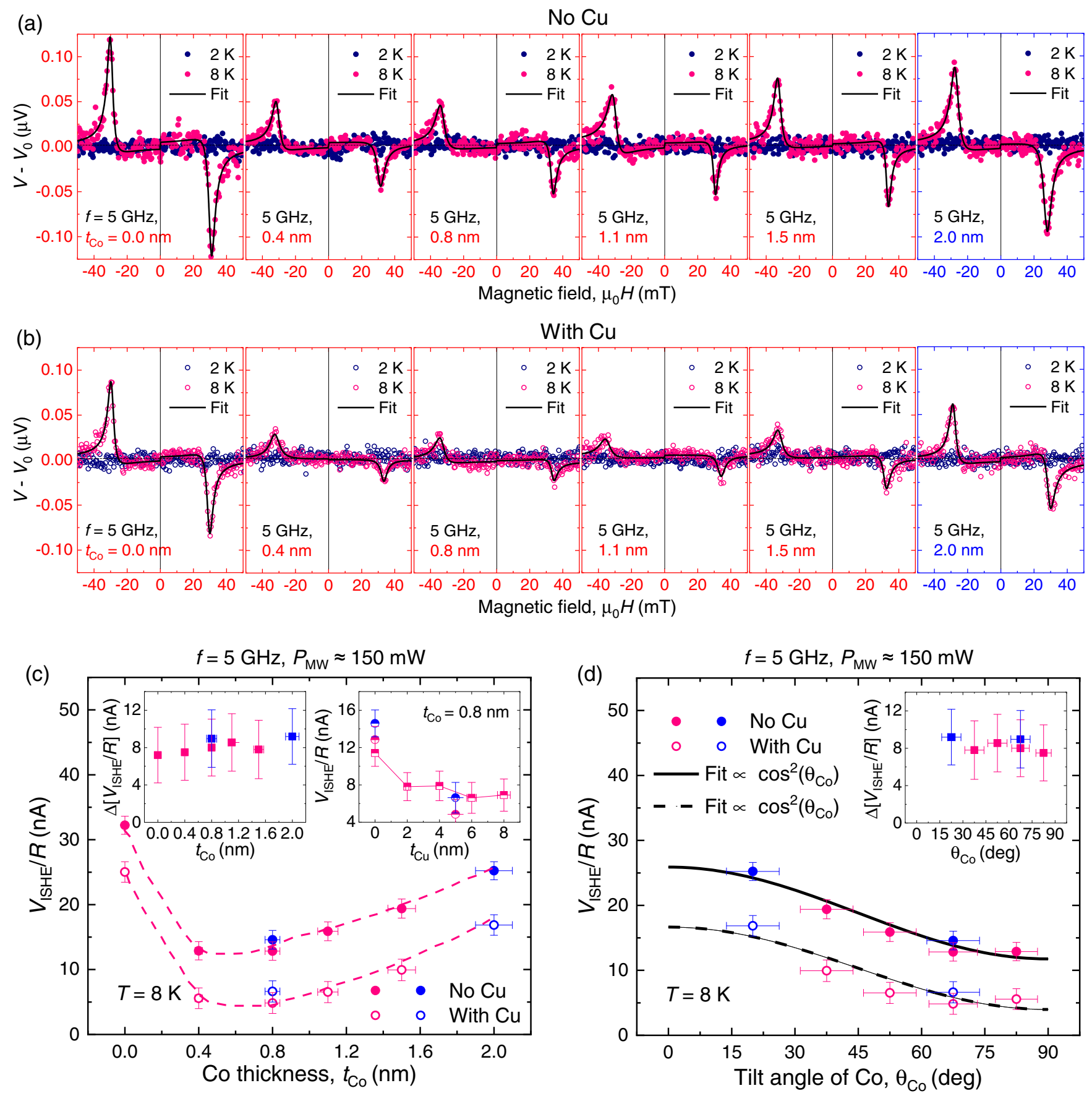

FIG. 3. Effect of $\mathrm{Cu}$ spacer addition and Co tilt angle on normal spin-transport properties. (a) Transverse dc voltage measurements for asymmetric $\mathrm{Pt}(2.0 \mathrm{~nm}) / \mathrm{Co}\left(t_{\mathrm{Co}}\right) / \mathrm{Pt}(1.7 \mathrm{~nm}) / \mathrm{Nb}(30 \mathrm{~nm}) / \mathrm{Ni}_{8} \mathrm{Fe}_{2}(6 \mathrm{~nm})$ samples with various Co thicknesses $t_{\mathrm{Co}}$ at a fixed microwave frequency $f=5 \mathrm{GHz}$, taken above and below $T_{c}$ of the couple $\mathrm{Nb}$. The black solid lines are fits to Lorentzian functions (see the Appendix). (b) Data equivalent to (a) but for asymmetric $\operatorname{Pt}(2.0 \mathrm{~nm}) / \mathrm{Co}\left(t_{\mathrm{Co}}\right) / \mathrm{Pt}(1.7 \mathrm{~nm}) / \mathrm{Cu}(5 \mathrm{~nm}) / \mathrm{Nb}(30 \mathrm{~nm}) / \mathrm{Ni}_{8} \mathrm{Fe}_{2}(6 \mathrm{~nm})$ samples. Inverse spin Hall effect (ISHE) voltage divided by the sample's resistance $V_{\text {ISHE }} / R$ as a function of $t_{\mathrm{Co}}$ (c) and the (effective) Co tilt angle $\theta_{\mathrm{Co}}$ (d) at $f=5 \mathrm{GHz}$. The dashed lines are guide to the eyes. The left (right) inset in (c) [(d)] shows the signal difference caused by the $\mathrm{Cu}(5 \mathrm{~nm})$ spacer addition whereas the right inset in (c) exhibits the $\mathrm{Cu}$ spacer thickness $t_{\mathrm{Cu}}$ dependence of ISHE for the $t_{\mathrm{Co}}=0.8 \mathrm{~nm}$ samples (see Supplemental Material, Sec. V, for details [17]). The dashed lines in (c) are guide to the eyes, whereas the black solid (dashed) line in (d) is a fit to $\cos ^{2}\left(\theta_{\mathrm{Co}}\right)$ for the $\mathrm{Cu}$-absent (Cu-present) samples. The red and blue symbols in (c) and (d) represent independent sets of the samples grown each in a single deposition run.

dependence of spin-pumping-induced inverse spin Hall effect (ISHE) [37,38] for the additional sets of asymmetric $\mathrm{Pt} / \mathrm{Co} / \mathrm{Pt} / \mathrm{Nb} / \mathrm{Ni}_{8} \mathrm{Fe}_{2}$ structures with and without $\mathrm{Cu}$ spacers (Fig. 6). This provides direct evidence for spin transport in the normal state. Figure 3(a) [Fig. 3(b)] displays the transverse dc voltage signals versus external 
(a)
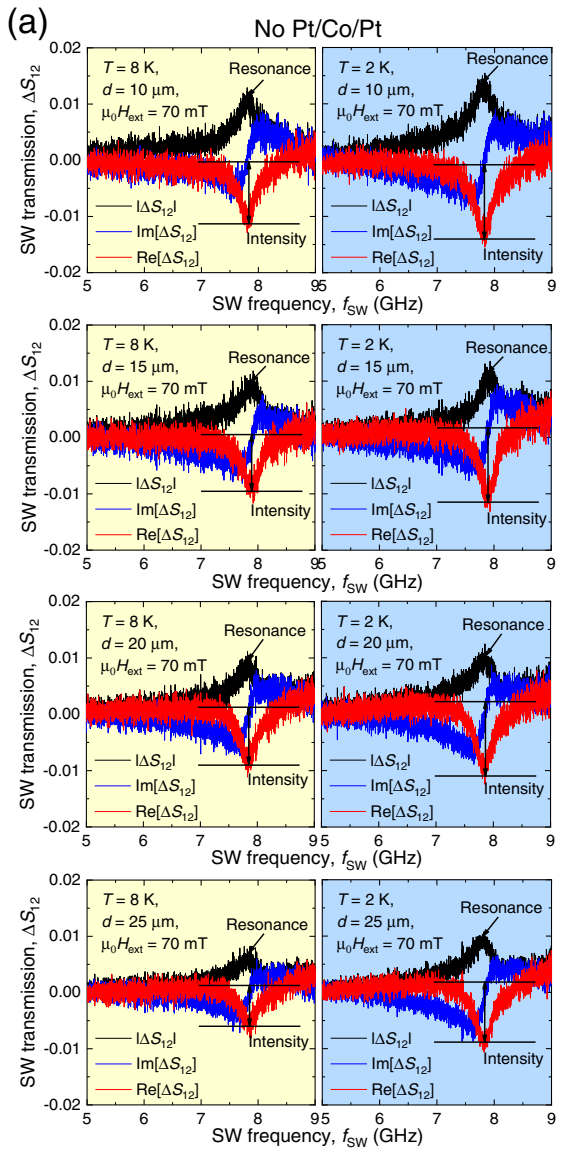

(b)
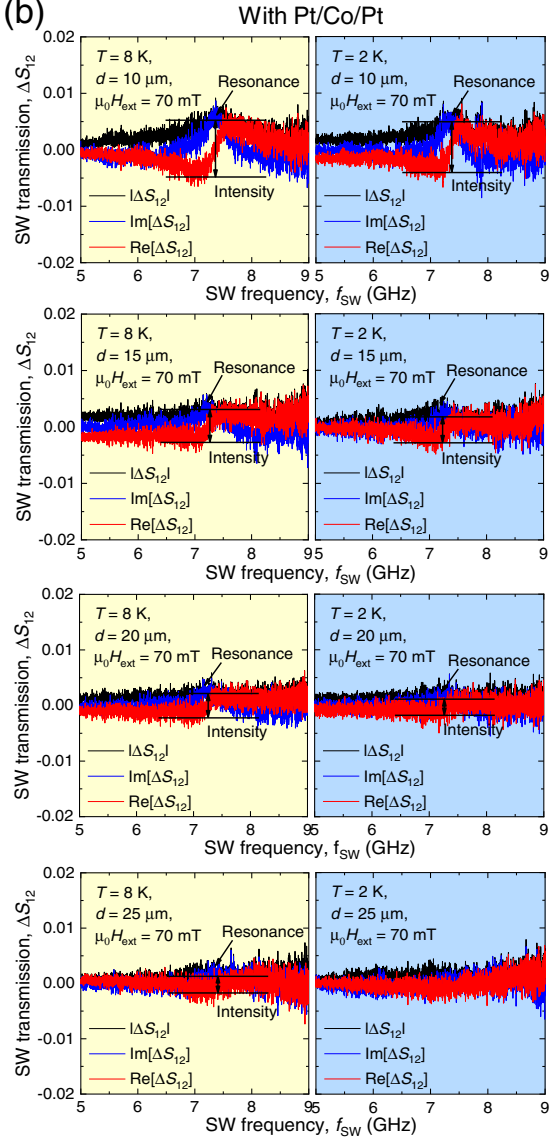

(c)

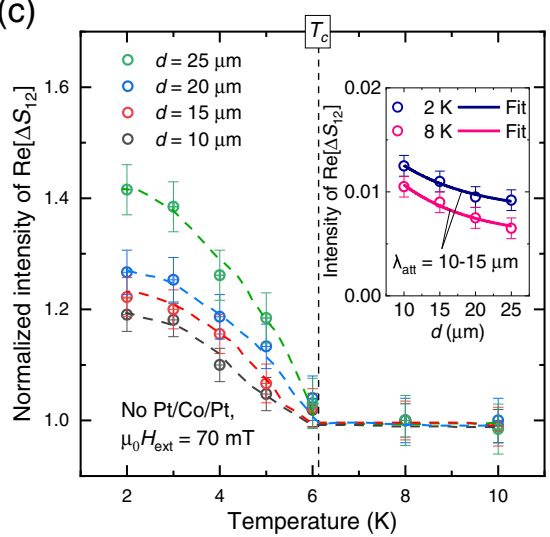

(d)

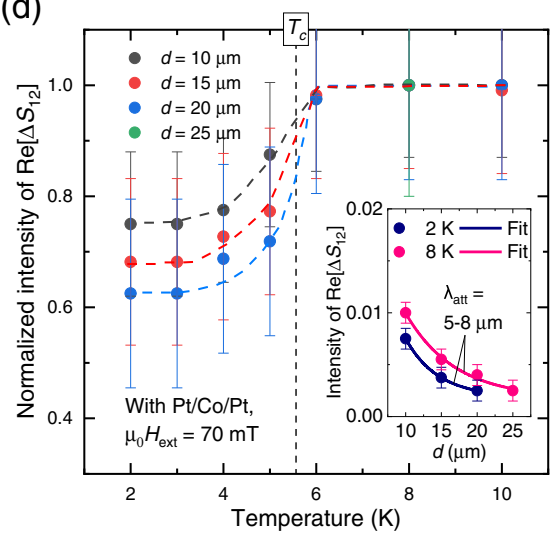

FIG. 4. Experimental realization of superconductor spin-wave (SW) devices. (a) Spin-wave transmission $\Delta S_{12}$ as a function of frequency $f$ for the $\mathrm{Nb}(30 \mathrm{~nm}) / \mathrm{Ni}_{8} \mathrm{Fe}_{2}(6 \mathrm{~nm}) / \mathrm{Nb}(30 \mathrm{~nm})$ device with a different distance $d(10-25 \mu \mathrm{m})$ between two separate antennas. These spectra are obtained under application of a fixed external magnetic field $\mu_{0} H=70 \mathrm{mT}$ above and below $T_{c}$ of the coupled $\mathrm{Nb}$. In each panel, the red, blue, and black curves represent, respectively, the real, imaginary, and absolute of $\Delta S_{12}$. (b) Data equivalent to (a) but for the $\mathrm{Pt}(2.0 \mathrm{~nm}) / \mathrm{Co}(0.8 \mathrm{~nm}) / \mathrm{Pt}(1.7 \mathrm{~nm}) / \mathrm{Nb}(30 \mathrm{~nm}) / \mathrm{Ni}_{8} \mathrm{Fe}_{2}(6 \mathrm{~nm}) / \mathrm{Nb}(30 \mathrm{~nm}) / \mathrm{Pt}(1.7 \mathrm{~nm}) / \mathrm{Co}(0.8 \mathrm{~nm}) /$ $\mathrm{Pt}(2.0 \mathrm{~nm})$ device. (c) Normalized intensity of the real part of $\Delta S_{12}$ across $T_{c}$ for the $\mathrm{Pt} / \mathrm{Co}(0.8 \mathrm{~nm}) / \mathrm{Pt}$-absent device with $d=10-25 \mu \mathrm{m}$. (d) Data equivalent to (c) but for the $\mathrm{Pt} / \mathrm{Co}(0.8 \mathrm{~nm}) / \mathrm{Pt}$-present device. Each inset shows the associated $d$ dependence of the signal intensity above and below $T_{c}$. The dashed lines in (c) and (d) are guide to the eyes whereas the solid lines in each inset are fits to an exponential decay function to estimate the SW attenuation length $\lambda_{\text {att }}[40,41]$ (see the Appendix).

IP $\mu_{0} H$ for the $\mathrm{Cu}$-absent (Cu-present) samples at $f=5 \mathrm{GHz}$, taken above and below $T_{c}$ (see the Appendix). Under IP FMR of the $\mathrm{Ni}_{8} \mathrm{Fe}_{2}$, a clear Lorentzian peak emerges in the dc voltage only in the normal state for both sample sets, which can be explained [38] by the strong decay of the quasiparticle charge-imbalance relaxation time immediately below $T_{c}$. Importantly, the polarity of the Lorentzian peak is identical (opposite) to that of $\mathrm{Pt} / \mathrm{Ni}_{8} \mathrm{Fe}_{2}\left(\mathrm{Nb} / \mathrm{Ni}_{8} \mathrm{Fe}_{2}\right)$ bilayers [38], where the $\mathrm{Pt}(\mathrm{Nb})$ spin sink is known to have a positive (negative) spin Hall angle $\theta_{\mathrm{SH}}[20,38]$. This indicates that the pumped spin currents from the precessing $\mathrm{Ni}_{8} \mathrm{Fe}_{2}$ pass through the $\mathrm{Nb}(30 \mathrm{~nm})$ layer to a large extent to the $(\mathrm{Cu}) / \mathrm{Pt} / \mathrm{Co} / \mathrm{Pt}$ spin sinks and the overall ISHE in our structures is dominated by the $(\mathrm{Cu}) / \mathrm{Pt} / \mathrm{Co} / \mathrm{Pt}$ (rather than the $\mathrm{Nb}$ ).

For a quantitative analysis, we plot the ISHE voltage divided by sample resistance $V_{\text {ISHE }} / R$ versus $t_{\text {Co }}$ [Fig. 3(c)] and $\theta_{\mathrm{Co}}$ [Fig. 3(d)]. In these plots, we can see that there is a clear decrease in the ISHE signal by the addition of $\mathrm{Cu}$ and its magnitude is strongly $\theta_{\mathrm{Co}}$ dependent, which can be described by the rapid spin precession and dephasing of transverse spins [39] around $h_{\mathrm{ex}}$ of the Co layer: $\cos ^{2}\left(\theta_{\mathrm{Co}}\right)$ [black lines in Fig. 3(d)]. Note that the signal difference caused by the addition of $5 \mathrm{~nm}$ of $\mathrm{Cu}$ [insets in Figs. 3(c) and $3(\mathrm{~d})]$ is nearly $\theta_{\mathrm{Co}}$ independent. These results taken together support our argument that $\mathrm{Cu}$ spacers weaken the interfacial SOC strength and it is the Co tilt state that then plays a dominant role in the spin transport process.

Finally, we progress to show the potential to harness these effects in a proof-of-principle prototype SC-based spin-wave (SW) device (Fig. 4). The idea behind this is that lateral SW propagation $[40,41]$ in our proximity-engineered structure (e.g., $\alpha_{2 K}-\alpha_{8 K} \approx 0.005$ for the $t_{C o}=$ $0.8 \mathrm{~nm}$ sample) between microwave injector and detector 
antennas is readily altered by opening or closing the vertical spin transport channel via the proximity creation of triplet pairing. Figures 4(a) and 4(b) show the $f$-dependent SW transmission $\Delta S_{12}$ of two types of the SW devices with and without $\mathrm{Pt} / \mathrm{Co}(0.8 \mathrm{~nm}) / \mathrm{Pt}$ spin sinks, obtained above and below $T_{c}$ at the fixed external IP $\mu_{0} H=70 \mathrm{mT}$ in the magnetostatic surface wave (MSSW) geometry [40,41] (see the Appendix and Sec. VI of Supplemental Material [17] for details). The observed spectra containing two major peaks in the low $f(<7 \mathrm{GHz})$ regime and satisfying the SW dispersion relationship (Videos 1-4 and Sec. VI in Supplemental Material [17]) and their exponential decay in the intensity with increasing the distance $d$ between the two separate antennas [Figs. 4(c) and 4(d)] indicate the propagating SWs [40,41]. Notably, the absence of characteristic dips [42] in the SW spectra (Videos 1-4 and Sec. VI of Supplemental Material [17]) indicates no significant nucleation or pinning of (OOP) Abrikosov vortices in our device structure.

The most noteworthy aspect in this demonstration is that on entering the superconducting state, the intensity of the lateral $\mathrm{SW}$ transmission signal rises (decays) when the $\mathrm{Pt} / \mathrm{Co}(0.8 \mathrm{~nm}) / \mathrm{Pt}$ spin sinks are removed (added) [Fig. 4(c)] [Fig. 4(d), and see also Videos 1-4 in Supplemental Material [17]], and the degree of this change becomes pronounced with increasing $d$. This is because SWs experience weaker (stronger) effective attenuation during lateral propagation if spin angular momentum is less (more) transmitted across the adjacent superconducting $\mathrm{Nb}$ to the spin loss regimes in the vertical direction. Note that the SW attenuation increases proportionally to the total FMR damping of the system [40,41].

With the $\mathrm{Pt} / \mathrm{Co}(0.8 \mathrm{~nm}) / \mathrm{Pt}$ spin sinks, we are able to modulate the lateral SW transmission intensity up to about $40 \%$ by proximity generating the vertical triplet spintransport channel. This result is encouraging and may provide a new type of SW logic functionality [43] activated in the superconducting state.

\section{CONCLUSIONS AND OUTLOOK}

We have shown that when a perpendicularly magnetized $\mathrm{Pt} / \mathrm{Co} / \mathrm{Pt}$ spin sink is proximity coupled to $\mathrm{Nb}$, superconducting spin-pumping efficiency can be tuned by controlling the effective $\theta_{\mathrm{Co}}$, i.e., by tuning the degree of orthogonality between the SOC and $h_{\mathrm{ex}}$ at the $\mathrm{Nb} / \mathrm{Pt} /$ $(\mathrm{Co} / \mathrm{Pt})$ interface $[4-6,8]$. We have also found that by comparison with the $\mathrm{Cu}$-present samples, the $\theta_{\mathrm{Co}}$-dependent superconducting spin-pumping efficiency reflects characteristic features of Rashba SOC-induced triplet pairing [4-6,8]. Our results provide a timely step toward understanding key interfacial properties for tuning superconducting spin transport mediated via equal-spin triplet states in a spin-singlet superconductor. The approach developed here can be used to explore and characterize triplet pair generation in SC-FM heterostructures with Rashba SOC by the application of superconducting charge currents and magnetic fields [44]. Our finely proximity-engineered structures enable experimental realization of a prototype SC-based SW device. This concept can be extended to any Rashba system [45,46] for the development of superconducting spin-logic devices [1] in which SOC is gate tunable [45], leading to a superconducting spin-based transistor.

The data used in this paper can be accessed here [47].

\section{ACKNOWLEDGMENTS}

This work was funded by the EPSRC Programme Grant "Superspin" (No. EP/N017242/1) and EPSRC International Network "Oxide Superspin" (No. EP/P026311/1). The work was in-part funded by a Leverhulme Trust Research Project Grant No. RPG-2016-306.

\section{APPENDIX: EXPERIMENTAL METHODS}

\section{Sample growth}

Symmetric $\mathrm{Pt} / \mathrm{Co} / \mathrm{Pt} / \mathrm{Nb} / \mathrm{Ni}_{8} \mathrm{Fe}_{2} / \mathrm{Nb} / \mathrm{Pt} / \mathrm{Co} / \mathrm{Pt}$ and asymmetric $\mathrm{Pt} / \mathrm{Co} / \mathrm{Pt} / \mathrm{Nb} / \mathrm{Ni}_{8} \mathrm{Fe}_{2}$ multilayers, with and without $\mathrm{Cu}$ spacer layers, were grown on $5 \times 5 \mathrm{~mm}^{2}$ thermally oxidized $\mathrm{Si}$ substrates by dc magnetron sputtering in an ultrahigh vacuum chamber $[9,19]$. The symmetric and asymmetric structures were prepared, respectively, for the ferromagnetic resonance (FMR) absorption $[9,19]$ and inverse spin Hall effect (ISHE) (or transverse dc voltage) [38] measurements. All layers were grown in situ at room temperature. $\mathrm{Ni}_{8} \mathrm{Fe}_{2}, \mathrm{Nb}, \mathrm{Co}$, and $\mathrm{Cu}$ are deposited at an $\mathrm{Ar}$ pressure of $1.5 \mathrm{~Pa}$ and $\mathrm{Pt}$ at $3.0 \mathrm{~Pa}$. The typical deposition rates were $5.1 \mathrm{~nm} / \mathrm{min}$ for $\mathrm{Ni}_{8} \mathrm{Fe}_{2}, 21.1 \mathrm{~nm} / \mathrm{min}$ for $\mathrm{Nb}$, $6.0 \mathrm{~nm} / \mathrm{min}$ for Co, $9.7 \mathrm{~nm} / \mathrm{min}$ for $\mathrm{Cu}$, and $7.6 \mathrm{~nm} / \mathrm{min}$ for Pt. The thicknesses of $\mathrm{Ni}_{8} \mathrm{Fe}_{2}, \mathrm{Nb}$, inner (outer) Pt, and $\mathrm{Cu}$ layers were kept constant at 6, 30, 1.7 (2.2), and $5 \mathrm{~nm}$, respectively, while the thickness of the Co layer varied from 0 to $2 \mathrm{~nm}$ to investigate the variation of FMR damping as a function of $t_{\mathrm{Co}}$ (or the Co tilt angle $\theta_{\mathrm{Co}}$ ) through the superconducting transition temperature $T_{c}$ of the coupled $\mathrm{Nb}$. Note that for all samples, the $\mathrm{Nb}$ (inner Pt) thickness is fixed at $30(1.7) \mathrm{nm}$ where the $\mathrm{Pt} / \mathrm{Co} / \mathrm{Pt}$ spin sink was proximity coupled through the $\mathrm{Nb}$ layer to the precessing $\mathrm{Ni}_{8} \mathrm{Fe}_{2}$ layer and the largest enhancement of spin pumping in the superconducting state was achieved in our prior FMR experiments $[9,19]$.

\section{Magnetization characterization}

The static magnetization hysteresis curves were measured on $5 \times 5 \mathrm{~mm}^{2}$ samples using a Quantum Design magnetic property measurement system at $8 \mathrm{~K}$, immediately above the superconducting transition temperature $T_{c}$. The external magnetic field was applied parallel and perpendicular to the film plane direction. Moreover, we carried out magnetic force microscopy (MFM) measurements on $\mathrm{Pt} / \mathrm{Co} / \mathrm{Pt} / \mathrm{Nb}$-only films (Fig. 5) to check local 
(a)

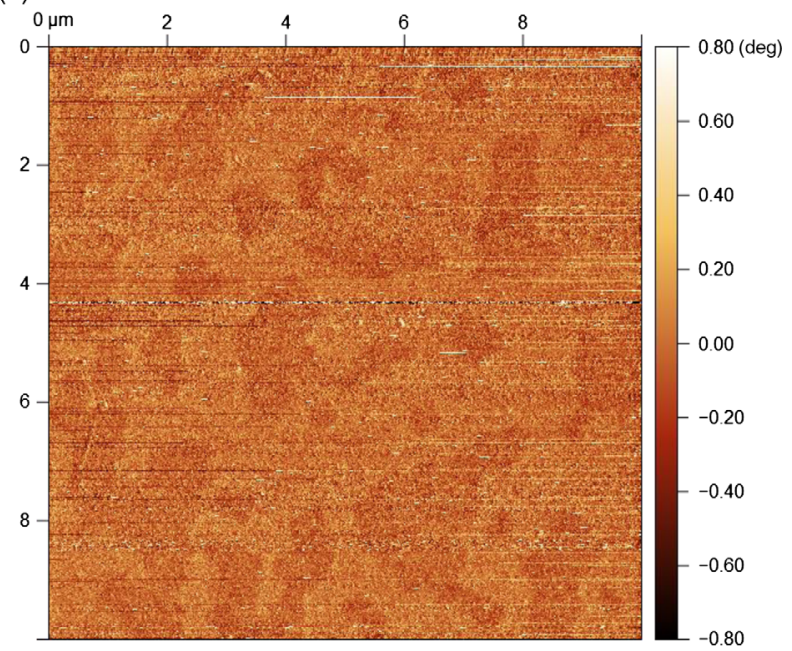

(b)

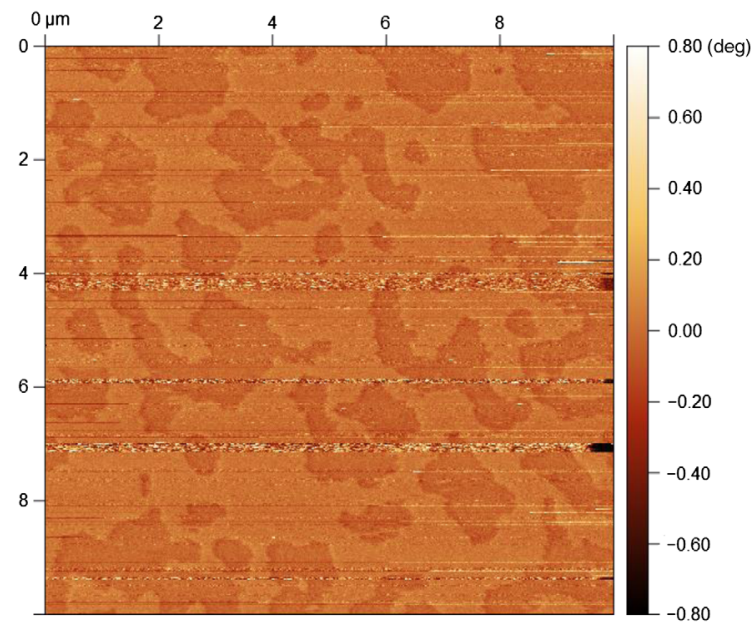

FIG. 5. Magnetic force microscopy (MFM) images of $\operatorname{Pt}(2.0 \mathrm{~nm}) / \mathrm{Co}(1.1 \mathrm{~nm}) / \mathrm{Pt}(1.7 \mathrm{~nm}) / \mathrm{Nb}(30 \mathrm{~nm})$ (a) and $\operatorname{Pt}(2.0 \mathrm{~nm}) /$ $\mathrm{Co}(1.5 \mathrm{~nm}) / \mathrm{Pt}(1.7 \mathrm{~nm}) / \mathrm{Nb}(30 \mathrm{~nm})(\mathrm{b})$, taken at the remanent state at $300 \mathrm{~K}$. The measured maze patterns of magnetic domains with a broad periodicity (a few microns) indicate a weak Dzyaloshinskii-Moriya interaction and the absence of magnetic Skyrmions in our $\mathrm{Pt} / \mathrm{Co} / \mathrm{Pt}$ symmetric structures, which are well consistent with previous experiments [22,23]. Note that for the Co thickness $t_{\mathrm{Co}}<1.1 \mathrm{~nm}$, the MFM contrast is below the noise level of our setup.

magnetic domain patterns and to assure the absence of a noncollinear magnetic ground state (e.g., magnetic Skrymion) [21-23]. For the microscopic characterization, we used a room temperature DI3100 magnetic force microscope operated under ambient conditions. We used a high moment $\mathrm{CoCr}$ cantilever (Bruker MESP-HM). For the measurement, we operated a dual mode (DI Lift mode) where the topography (not shown) was obtained in tapping mode and the MFM image (Fig. 5) was obtained at a tipsample distance of $30 \mathrm{~nm}$.

\section{Superconducting transition measurement}

dc electrical transport measurements were conducted on (unpatterned) $5 \times 5 \mathrm{~mm}^{2}$ samples using a custom-built dipstick probe in a liquid helium Dewar with a four-point current-voltage method. The resistance $R$ (of a sample) versus temperature $T$ curves were obtained at the applied current $I$ of $\leq 0.1 \mathrm{~mA}$ while decreasing $T$. From the $T$ derivative of $R, d R / d T, T_{c}$ was defined as the $T$ value that exhibits the maximum of $d R / d T$.

We analyzed our $T_{c}\left(t_{\mathrm{Co}}\right)$ data [Fig. 1(d)] using the following approximate formula [26]:

$$
\begin{aligned}
& \ln \left[\frac{T_{c}}{T_{c}^{*}}\right] \\
& \quad \approx \Psi\left(\frac{1}{2}\right)-\operatorname{Re}\left\{\Psi\left[\frac{1}{2}+\frac{2 T_{c}^{*}}{T_{c} \tilde{\tau}_{0}} \frac{1}{\tilde{\gamma}+\frac{1-i}{2} \operatorname{coth}\left[(1+i)\left(\frac{t_{\mathrm{FM}}}{2 \xi_{\mathrm{FM}}}\right)\right]}\right]\right\},
\end{aligned}
$$

where $T_{c}^{*}=T_{c}\left(t_{F M}=0\right), \Psi$ is the digamma function, $\tilde{\tau}_{0}^{-1}=\left(1 / 4 \pi T_{c}^{*}\right)\left(D_{\mathrm{SC}} / t_{\mathrm{SC}} \xi_{\mathrm{FM}}\right)\left(\rho_{\mathrm{SC}} / \rho_{\mathrm{FM}}\right), D_{\mathrm{SC}}$ is the diffusion coefficient of the $\mathrm{Nb}\left(10 \mathrm{~cm}^{2} / \mathrm{s}\right.$ at $\left.8 \mathrm{~K}\right), t_{\mathrm{SC}}$ is the $\mathrm{Nb}$ thickness $(30 \mathrm{~nm})$, and $\rho_{\mathrm{SC}}\left(\rho_{\mathrm{FM}}\right)$ is the conductivity of the
$\mathrm{Nb}(\mathrm{Co})[7(30) \mu \Omega \mathrm{cm}$ at $8 \mathrm{~K}] \cdot \tilde{\gamma}=\gamma_{B}\left(\xi_{\mathrm{SC}} / \xi_{\mathrm{FM}}\right)$, where $\gamma_{B}$ is the interface transparency and $\xi_{\mathrm{SC}}$ is the (dirty-limit) coherence length of the $\mathrm{Nb}(16-18 \mathrm{~nm}$ at $2 \mathrm{~K}$ ) [9]. Note that in this formula, only the influence of $h_{\mathrm{ex}}$ on the order parameter is taken into account [26].

When the out-of-plane (OOP) component of magnetic fluxes (e.g., stray fields from the OOP-magnetized Co layer) exists, unintentional Abrikosov vortex nucleation [48] can suppress the pair potential, the superconducting volume, and thus the singlet Cooper pair density of $\mathrm{Nb}$ that is the underlying source of proximity-induced triplet pairing. To take this detrimental effect into account in Figs. 2(e) and 2(f), we normalized the damping difference across $T_{c}$ by the calculated superconducting gap $2 \Delta$ at $2 \mathrm{~K}$ from the measured $T_{c}$ data [Fig. 1(d)], which is directly proportional to the singlet pair density:

$$
\Delta(T) \approx 1.76 k_{B} T_{c} \tanh \left[1.74 \sqrt{1-\frac{T}{T_{c}}}\right],
$$

where $k_{B}$ is Boltzmann's constant.

\section{Broadband FMR absorption and ISHE measurements}

We measured the FMR response of the sample attached on a broadband coplanar waveguide (CPW) with either dc field or rf pulse modulation $[9,19]$. To obtain each FMR spectrum, the microwave power absorbed by the sample was measured while sweeping the external static magnetic field $\mu_{0} H$ at the fixed microwave frequency $f$ of 5-20 GHz. At the beginning of each measurement, we applied a large IP $\mu_{0} H(0.5 \mathrm{~T})$ to fully magnetize the $\mathrm{Ni}_{8} \mathrm{Fe}_{2}$ layer, after 
which the field was reduced to the range of FMR. Once the $f$-dependent FMR measurements (from high to low $f$ ) were complete, the field was returned to zero to cool the system down further for a lower $T$ measurement. For all FMR absorption measurements, the microwave (MW) power was set to $10 \mathrm{dBm}$ where the actual microwave power absorbed in the sample is a few milliwatt that has no measurable effect on $T_{c}$ of the Nb layer [9]. Based on our previous ISHE experiment (Fig. S1 of Ref. [38]), it is reasonable to assume that unintentional heating at a higher power ( $\geq 50 \mathrm{~mW}$ in our setup) reduces profoundly the real superconducting volume, the effective pair potential, and thus the overall singlet pair density of $\mathrm{Nb}$ layers, which is the underlying source of proximity-induced triplet pairing. Note also that the fixed thickness $(30 \mathrm{~nm})$ of $\mathrm{Nb}$ layers studied here is much less than the magnetic penetration depth in the superconducting state $(\geq 100 \mathrm{~nm}$ in thin $\mathrm{Nb}$ films), and so there is no considerable effect of Meissner screening on the local (dc or rf) magnetic field experienced by $\mathrm{Ni}_{8} \mathrm{Fe}_{2}$ below $T_{c}$, as supported by the insensitivity of the resonance field $\mu_{0} H_{\text {res }}$ across $T_{c}$ [Figs. 2(a) and 2(b)]. We employed a vector field cryostat from Cryogenic Ltd. that can apply a $1.2 \mathrm{~T}$ magnetic field in any direction over a $T$ range of $2-300 \mathrm{~K}$.

We first fitted the FMR absorption data (Sec. III of Supplemental Material [17]) with the field derivative of symmetric and antisymmetric Lorentzian functions [32] to accurately determine the FMR linewidth $\mu_{0} \Delta H$ and the resonance field $\mu_{0} H_{\text {res }}$ :

$$
\begin{aligned}
\frac{d \chi^{\prime \prime}}{d H} \propto & A\left[\frac{\left(\Delta H_{\mathrm{HWHM}}\right)^{2}\left(H-H_{\mathrm{res}}\right)}{\left[\left(\Delta H_{\mathrm{HWHM}}\right)^{2}+\left(H-H_{\mathrm{res}}\right)^{2}\right]^{2}}\right] \\
& +B\left[\frac{\left(\Delta H_{\mathrm{HWHM}}\right)\left(H-H_{\mathrm{res}}\right)^{2}-\left(\Delta H_{\mathrm{HWHM}}\right)^{3}}{\left[\left(\Delta H_{\mathrm{HWHM}}\right)^{2}+\left(H-H_{\mathrm{res}}\right)^{2}\right]^{2}}\right],
\end{aligned}
$$

where $A(B)$ is the amplitude of the field derivative of the symmetric (antisymmetric) Lorentzian function, $\mu_{0} H$ is the external dc magnetic field, and $\mu_{0} \Delta H_{\mathrm{HWHM}}=$ $(\sqrt{3} / 2) \mu_{0} \Delta H$ is the half width at half maximum (HWHM) of the imaginary part $\chi^{\prime \prime}$ of the magnetic susceptibility.

From the linear scaling of $\mu_{0} \Delta H$ with $f$ [Figs. 2(a) and 2(b)], we calculated the effective Gilbert(-type) damping constant $\alpha: \mu_{0} \Delta H(f)=\mu_{0} \Delta H_{0}+(4 \pi \alpha f / \sqrt{3} \gamma)$, where $\mu_{0} \Delta H_{0}$ is the zero-frequency line broadening. We also estimated the effective saturation magnetization $\mu_{0} M_{s}$ (of the $\mathrm{Ni}_{8} \mathrm{Fe}_{2}$ ) from the dispersion relation of $\mu_{0} H_{\text {res }}$ with $f$ [inset of Figs. 2(a) and 2(b)] using Kittel's formula, $f=(\gamma / 2 \pi) \sqrt{\left[\mu_{0}\left(H_{\text {res }}+M_{\text {eff }}\right) \mu_{0} H_{\text {res }}\right]}$, where $\gamma=g_{L} \mu_{B} / \hbar$ is the gyromagnetic ratio $\left(1.84 \times 10^{11} \mathrm{~T}^{-1} \mathrm{~s}^{-1}\right), g_{L}$ is the Landé $g$ factor (taken to be 2.1), $\mu_{B}$ is the Bohr magneton, and $\hbar$ is Plank's constant divided by $2 \pi$.

For the ISHE (or transverse dc voltage) measurement (Fig. 6) [38], the sample was attached face down on the CPW by using an electrically insulating high-vacuum

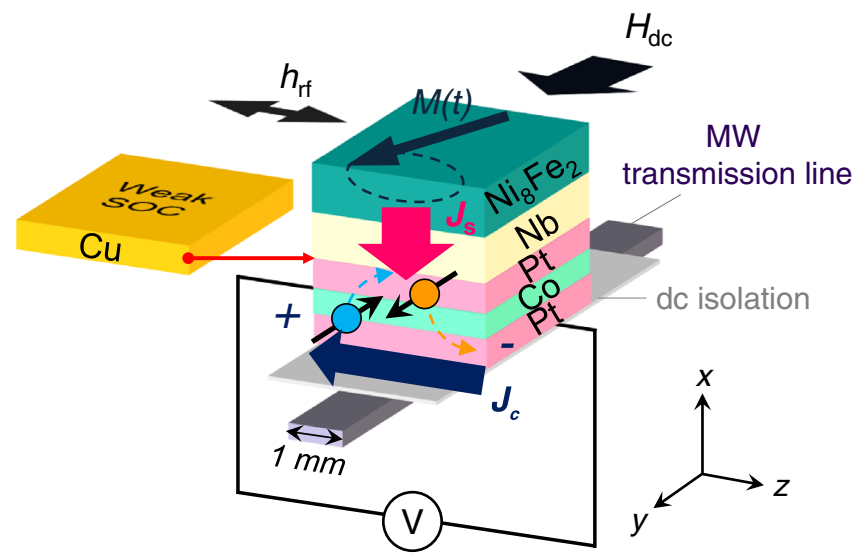

FIG. 6. Measurement scheme of the spin-pumping-induced inverse spin Hall effect (ISHE). A dynamically injected spin current $J_{s}$ from precessing $\mathrm{Ni}_{8} \mathrm{Fe}_{2}$ through $\mathrm{Nb}$ into $\mathrm{Pt} / \mathrm{Co} / \mathrm{Pt}$ spin sink is converted into a transverse charge current $J_{c}$ via the ISHE (mostly in the $\mathrm{Pt} / \mathrm{Co} / \mathrm{Pt}$ spin sink), producing a measurable electromotive force $V$.

grease. A microwave signal was passed through the CPW and excited FMR of the $\mathrm{Ni}_{8} \mathrm{Fe}_{2}$ layer; a transverse dc voltage as a function of $\mu_{0} H$ was measured between two Ag-paste contacts at opposite ends of the sample. In these measurements, the microwave frequency was fixed at $5 \mathrm{GHz}$ and the microwave power at the CPW at approximately $150 \mathrm{~mW}$ (for $T=2$ and $8 \mathrm{~K}$ ), which yields measurable signals ( $\geq 5 \mathrm{nV}$ ) in our setup.

The measured dc voltage [Figs. 3(a) and 3(b)] can be decomposed into symmetric and antisymmetric Lorentzian functions with respect to $\mu_{0} H_{\text {res }}$, with weights of $V_{\text {sym }}$ and $V_{\text {asy }}$, respectively [38]:

$$
\begin{aligned}
V(H)-V_{0}= & V_{\text {sym }}\left[\frac{\left(\Delta H^{\prime}\right)^{2}}{\left(\Delta H^{\prime}\right)^{2}+\left(H-H_{\text {res }}\right)^{2}}\right] \\
& +V_{\text {asy }}\left[\frac{\left(\Delta H^{\prime}\right)\left(H-H_{\text {res }}\right)}{\left(\Delta H^{\prime}\right)^{2}+\left(H-H_{\text {res }}\right)^{2}}\right],
\end{aligned}
$$

where $V_{0}$ is a background voltage and $\mu_{0} \Delta H^{\prime}$ is the HWHM of the dc voltage. We attributed $V_{\text {sym }}$ to the ISHE signal $V_{\text {ISHE }}$. If the Co thickness in the $\mathrm{Pt} / \mathrm{Co} / \mathrm{Pt}$ spin sink is larger than its spin dephasing length (a few angstroms) [39], $V_{\text {ISHE }}\left(\theta_{\text {Co }}\right)$ is simply proportional to $\cos ^{2}\left(\theta_{\mathrm{Co}}\right)[$ Fig. $3(\mathrm{~d})]$.

\section{SW device fabrication}

To fabricate the standard SW devices [40,41] displayed in Fig. 7 and Sec. VI of the Supplemental Material [17], the Hall bar(-type) structures with an active SW track of $50 \times$ $50 \mu \mathrm{m}^{2}$ were patterned into the in situ grown $\mathrm{Nb} /$ $\mathrm{Ni}_{8} \mathrm{Fe}_{2} / \mathrm{Nb}$ films with and without $\mathrm{Pt} / \mathrm{Co}(0.8 \mathrm{~nm}) / \mathrm{Pt}$ spin sinks by using optical lithography and Ar-ion beam etching. After depositing AlN (40 nm) for dc electrical isolation by reactive sputtering, coplanar waveguides 


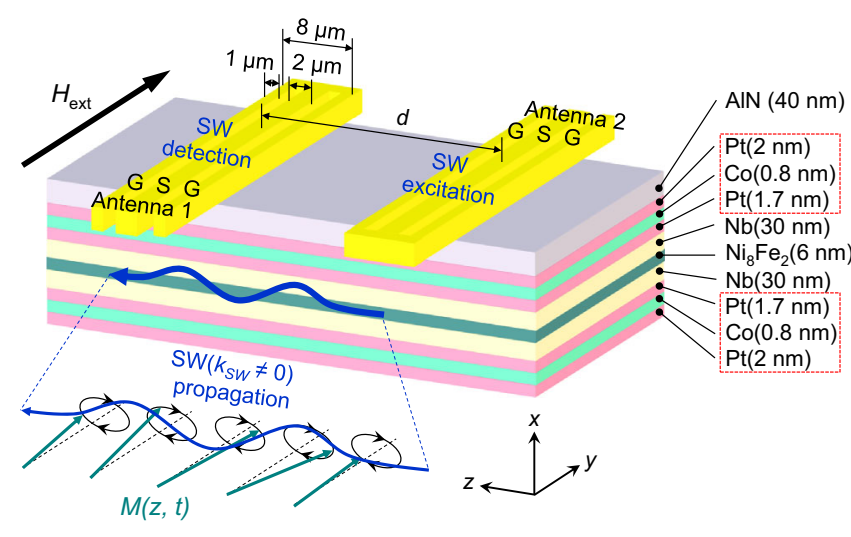

FIG. 7. Schematic illustration of propagating SW spectroscopy on the proximity-engineered structure with $\mathrm{Pt} / \mathrm{Co}(0.8 \mathrm{~nm}) / \mathrm{Pt}$ spin sinks. When the middle $\mathrm{Ni}_{8} \mathrm{Fe}_{2}$ layer is (in-plane) magnetized along the positive $y$ direction, the $\mathrm{SW}\left(k_{\mathrm{SW}} \neq 0\right)$ driven by the microwave excitation propagates along the positive $z$ direction via a collective precession of magnetization $M(z, t)$.

(CPWs or MW antennas) with various interspacing of 10-25 $\mu \mathrm{m}$ were patterned on top of the SW track using electron-beam lithography and lift-off of sputtered $\mathrm{Cu}(100 \mathrm{~nm}) / \mathrm{Ti}(5 \mathrm{~nm})$ layers. Two identical CPWs consist of a MW signal line ( $2 \mu \mathrm{m}$ wide) and two ground lines ( $1 \mu \mathrm{m}$ wide) with an intraseparation of $2 \mu \mathrm{m}$, which preferentially excites or detects the SWs with a wave number $k_{\mathrm{SW}}$ in the range of $0.9 \pm 0.6 \mu \mathrm{m}^{-1}$ (see Sec. VI of the Supplemental Material [17]).

\section{Propagating SW spectroscopy}

A pair of antennas of the SW device were connected to ports 1 and 2 of a vector network analyzer (VNA, Rohde \& Schwarz, $100 \mathrm{MHz}-20 \mathrm{GHz}$ ) by multiple wire bonding to a precalibrated sample holder (having the $50 \Omega$ impedance) via phase-stable coaxial cables. The $f$-dependent forward complex transmission coefficient (e.g., scattering parameter $S_{12}$; the MW power received at port 1 relative to the power conveyed to port 2) was measured in the variable temperature insert of a vector field cryostat by applying a fixed external IP $\mu_{0} H$ transverse to the SW propagation direction (or wave vector $\boldsymbol{k}_{\mathrm{SW}}$ ), the so-called MSSW configuration $[40,41]$. The input MW power was set to $0 \mathrm{dBm}$ (the actual power delivered to the device in our setup was $<100 \mu \mathrm{W}$ ), so that the nonlinear response of magnetization dynamics and the unintentional heating effect on the $\mathrm{Nb}$ layer can be avoided. The SW transmission signal $\Delta S_{12}\left(f, \mu_{0} H\right)$ of interest was analyzed by subtracting the nonmagnetic background $S_{12}\left(f, \mu_{0} H_{\text {ref }}\right)$ under application of a large reference field $\mu_{0} H_{\text {ref }}(0.12 \mathrm{~T})$ and normalizing the $f$ dependence $[40,41]$ :

$$
\Delta S_{12}\left(f, \mu_{0} H\right)=\frac{S_{12}\left(f, \mu_{0} H\right)-S_{12}\left(f, \mu_{0} H_{\text {ref }}\right)}{S_{12}\left(f, \mu_{0} H_{\text {ref }}\right)} .
$$

The SW dispersion in the MSSW mode for symmetric sample structures is given by $[40,41]$

$$
f_{\mathrm{SW}} \approx \frac{\gamma}{2 \pi} \sqrt{\left[\mu_{0}\left(H_{\mathrm{res}}+M_{\mathrm{eff}}\right) \mu_{0} H_{\mathrm{res}}+\left(\frac{\mu_{0} M_{\mathrm{eff}}}{2}\right)^{2}\left[1-\exp \left(-2 k_{\mathrm{SW}} t\right)\right]\right.},
$$

where $t$ is the $\mathrm{Ni}_{8} \mathrm{Fe}_{2}$ thickness $(6 \mathrm{~nm})$. By fitting the $\mathrm{SW}$ resonance, corresponding to the peak in the absolute of $\Delta S_{12}$ [ $=\left|\Delta S_{12}\right|$, Figs. 4(a) and 4(b)], to this dispersion relationship, we extracted the $k_{\mathrm{SW}}$ and $\mu_{0} M_{\text {eff }}$ values (Sec. VI of Supplemental Material [17]) for the $\mathrm{Ni}_{8} \mathrm{Fe}_{2}$ layer. In addition, we deduced the SW attenuation length $\lambda_{\text {SW }}$ [insets of Figs. 4(c) and 4(d)] from the fact $[40,41]$ that the SW intensity, defined as the maximum peak-to-valley height of the real part of $\Delta S_{12}\left[=\operatorname{Re}\left[\Delta S_{12}\right]\right.$, Figs. 4(a) and 4(b)], exponentially decays with increasing $d$ : $\exp \left[-\left(d / \lambda_{\mathrm{SW}}\right)\right]$. Here, $\lambda_{\mathrm{SW}}=v_{g} \tau_{\text {pres }}, v_{g}=2 \pi\left(\partial f_{\mathrm{SW}} / \partial k_{\mathrm{SW}}\right)$ is the group velocity, and $\tau_{\text {pres }}=\left\{\alpha \gamma\left[\mu_{0}\left(2 H_{\text {res }}+M_{\text {eff }}\right)\right]\right\}^{-1}$ is the magnetization precession time.

[1] J. Linder and J. W. A. Robinson, Superconducting Spintronics, Nat. Phys. 11, 307 (2015).
[2] M. Eschrig, Spin-Polarized Supercurrents for Spintronics: A Review of Current Progress, Rep. Prog. Phys. 78, 104501 (2015).

[3] N. O. Birge, Spin-Triplet Supercurrents in Josephson Junctions Containing Strong Ferromagnetic Materials, Phil. Trans. R. Soc. A 376, 20150150 (2018).

[4] F. S. Bergeret and I. V. Tokatly, Spin-Orbit Coupling as a Source of Long-Range Triplet Proximity Effect in Superconductor-Ferromagnet Hybrid Structures, Phys. Rev. B 89, 134517 (2014).

[5] S. H. Jacobsen, J. A. Ouassou, and J. Linder, Critical Temperature and Tunneling Spectroscopy of Superconductor-Ferromagnet Hybrids with Intrinsic Rashba-Dresselhaus Spin-Orbit Coupling, Phys. Rev. B 92, 024510 (2015).

[6] S. H. Jacobsen, I. Kulagina, and J. Linder, Controlling Superconducting Spin Flow with Spin-Flip Immunity Using a Single Homogeneous Ferromagnet, Sci. Rep. 6, 23926 (2016).

[7] X. Montiel and M. Eschrig, Generation of Pure Superconducting Spin Current in Magnetic Heterostructures via 
Nonlocally Induced Magnetism due to Landau Fermi Liquid Effects, Phys. Rev. B 98, 104513 (2018).

[8] M. Amundsen and J. Linder, Quasiclassical Theory for Interfaces with Spin-Orbit Coupling, Phys. Rev. B 100, 064502 (2019).

[9] K.-R. Jeon, C. Ciccarelli, A. J. Ferguson, H. Kurebayashi, L. F. Cohen, X. Montiel, M. Eschrig, J. W. A. Robinson, and M. G. Blamire, Enhanced Spin Pumping into Superconductors Provides Evidence for Superconducting Pure Spin Currents, Nat. Mater. 17, 499 (2018).

[10] N. Banerjee, J. A. Ouassou, Y. Zhu, N. A. Stelmashenko, J. Linder, and M. G. Blamire, Controlling the Superconducting Transition by Spin-Orbit Coupling, Phys. Rev. B 97, 184521 (2018).

[11] N. Satchell and N. O. Birge, Supercurrent in Ferromagnetic Josephson Junctions with Heavy Metal Interlayers, Phys. Rev. B 97, 214509 (2018).

[12] N. Satchell, R. Loloee, and N. O. Birge, Supercurrent in Ferromagnetic Josephson Junctions with Heavy Metal Interlayers II: Canted Magnetization, Phys. Rev. B 99, 174519 (2019).

[13] I. Martínez, P Högl, C. González-Ruano, J. P. Cascales, C. Tiusan, Y. Lu, M. Hehn, A. Matos-Abiague, J. Fabian, I. Žutić, and F. G. Aliev, Interfacial Spin-Orbit Coupling: New Platform for Superconducting Spintronics, Phys. Rev. Applied, 13, 014030 (2020).

[14] Y. Tserkovnyak, A. Brataas, G. E. W. Bauer, and B. I. Halperin, Nonlocal Magnetization Dynamics in Ferromagnetic Heterostructures, Rev. Mod. Phys. 77, 1375 (2005).

[15] N. Nakajima, T. Koide, T. Shidara, H. Miyauchi, H. Fukutani, A. Fujimori, K. Iio, T. Katayama, M. Nývlt, and Y. Suzuki, Perpendicular Magnetic Anisotropy Caused by Interfacial Hybridization via Enhanced Orbital Moment in Co/Pt Multilayers: Magnetic Circular X-Ray Dichroism Study, Phys. Rev. Lett. 81, 5229 (1998).

[16] M. T. Johnson, P. J. H. Bloemen, F. J. A. Den Broeder, and J. J. De Vries, Magnetic Anisotropy in Metallic Multilayers, Rep. Prog. Phys. 59, 1409 (1996).

[17] See Supplemental Material at http://link.aps.org/ supplemental/10.1103/PhysRevX.10.031020 for analytic description of the Co tilt angle dependence of equal-spin triplet pairing, numerical calculation based on the Usadel formalism, FMR spectra for $\mathrm{Pt} / \mathrm{Co} / \mathrm{Pt} / \mathrm{Nb} / \mathrm{Ni}_{8} \mathrm{Fe}_{2} / \mathrm{Nb} / \mathrm{Pt} /$ $\mathrm{Co} / \mathrm{Pt}$ samples with and without $\mathrm{Cu}$ spacers, $\mathrm{Cu}$ spacer thickness dependence of superconducting spin-pumping efficiency, $\mathrm{Cu}$ spacer thickness dependence of normal spin-transport properties, experimental realization of superconducting spin-wave devices with $\mathrm{Pt} / \mathrm{Co}(0.8 \mathrm{~nm}) / \mathrm{Pt}$ spin sinks, and static magnetic properties of $\mathrm{Pt} / \mathrm{Co} / \mathrm{Pt}$ trilayers with and without the presence of a $\mathrm{Cu}$ spacer.

[18] C. Klewe, T. Kuschel, J.-M. Schmalhorst, F. Bertram, O. Kuschel, J. Wollschläger, J. Strempfer, M. Meinert, and G. Reiss, Static Magnetic Proximity Effect in $\mathrm{Pt} / \mathrm{Ni}_{1-x} \mathrm{Fe}_{x}$ Bilayers Investigated by X-Ray Resonant Magnetic Reflectivity, Phys. Rev. B 93, 214440 (2016).

[19] K.-R. Jeon, C. Ciccarelli, H. Kurebayashi, L. F. Cohen, X. Montiel, M. Eschrig, S. Komori, J. W. A. Robinson, and M. G. Blamire, Exchange-Field Enhancement of Superconducting Spin Pumping, Phys. Rev. B 99, 024507 (2019).
[20] T. Tanaka, H. Kontani, M. Naito, T. Naito, D. S. Hirashima, K. Yamada, and J. Inoue, Intrinsic Spin Hall Effect and Orbital Hall Effect in $4 d$ and $5 d$ Transition Metals, Phys. Rev. B 77, 165117 (2008).

[21] Y.-K. Park, D.-Y. Kim, J.-S. Kim, Y.-S. Nam, M.-H. Park, H.-C. Choi, B.-C. Min, and S.-B. Choe, Experimental Observation of the Correlation between the Interfacial Dzyaloshinskii-Moriya Interaction and Work Function in Metallic Magnetic Trilayers, NPG Asia Mater. 10, 995 (2018).

[22] M. Baćani, M. Marioni, J. Schwenk, and H. J. Hug, How to Measure the Local Dzyaloshinskii-Moriya Interaction in Skyrmion Thin-Film Multilayers, Sci. Rep. 9, 3114 (2019).

[23] C. Moreau-Luchaire, C. Moutafis, N. Reyren, J. Sampaio1, C. A. F. Vaz, N. Van Horne, K. Bouzehouane, K. Garcia, C. Deranlot, P. Warnicke, P. Wohlhüter, J.-M. George, M. Weigand, J. Raabe, V. Cros, and A. Fert, Additive Interfacial Chiral Interaction in Multilayers for Stabilization of Small Individual Skyrmions at Room Temperature, Nat. Nanotechnol. 11, 444 (2016).

[24] A. I. Larkin and Y. N. Ovchinnikov, Nonuniform State of Superconductors, Zh. Eksp. Teor. Fiz. 47, 1136 (1964) [Sov. Phys. JETP 20, 762 (1965)].

[25] P. Fulde and R. A. Ferrell, Superconductivity in a Strong Spin-Exchange Field, Phys. Rev. 135, A550 (1964).

[26] A. I. Buzdin, Proximity Effects in SuperconductorFerromagnet Heterostructures, Rev. Mod. Phys. 77, 935 (2005).

[27] J. Kim, J. H. Kwon, K. Char, H. Doh, and H.-Y. Choi, Quantitative Analysis of the Proximity Effect in $\mathrm{Nb} / \mathrm{Co}_{60} \mathrm{Fe}_{40}, \mathrm{Nb} / \mathrm{Ni}$, and $\mathrm{Nb} / \mathrm{Cu}_{40} \mathrm{Ni}_{60}$ Bilayers, Phys. Rev. B 72, 014518 (2005).

[28] K. Kim, J. H. Kwon, J. Kim, K. Char, H. Doh, and H.-Y. Choi, Proximity Effect in $\mathrm{Nb} / \mathrm{Cu} / \mathrm{CoFe}$ Trilayers, Phys. Rev. B 74, 174503 (2006).

[29] T. Y. Lee, Y. C. Won, D. S. Son, S. H. Lim, and S.-R. Lee, Strength of Perpendicular Magnetic Anisotropy at Bottom and Top Interfaces in $[\mathrm{Pt} / \mathrm{Co} / \mathrm{Pt}]$ Trilayers, IEEE Magn. Lett. 5, 1000104 (2014).

[30] P. J. Metaxas, J. P. Jamet, A. Mougin, M. Cormier, J. Ferré, V. Baltz, B. Rodmacq, B. Dieny, and R. L. Stamps, Creep and Flow Regimes of Magnetic Domain-Wall Motion in Ultrathin Pt/Co/Pt Films with Perpendicular Anisotropy, Phys. Rev. Lett. 99, 217208 (2007).

[31] A. Mougin, M. Cormier, J. P. Adam, P. J. Metaxas, and J. Ferré, Domain Wall Mobility, Stability and Walker Breakdown in Magnetic Nanowires, Europhys. Lett. 78, 57007 (2007).

[32] Z. Celinski, K. B. Urquhart, and B. Heinrich, Using Ferromagnetic Resonance to Measure the Magnetic Moments of Ultrathin Films, J. Magn. Magn. Mater. 166, 6 (1997).

[33] W. Zhang, W. Han, X. Jiang, S.-H. Yang, and S. S. P. Parkin, Role of Transparency of Platinum-Ferromagnet Interfaces in Determining the Intrinsic Magnitude of the Spin Hall Effect, Nat. Phys. 11, 496 (2015).

[34] J. Bass and W. P. Pratt, Jr., Spin-Diffusion Lengths in Metals and Alloys, and Spin-Flipping at Metal/Metal Interfaces: An Experimentalist's Critical Review, J. Phys. Condens. Matter 19, 183201 (2007). 
[35] Y. V. Fominov, A. A. Golubov, T. Yu. Karminskaya, M. Yu. Kupriyanov, R. G. Deminov, and L. R. Tagirov, Superconducting Triplet Spin Valve, JETP Lett. 91, 308 (2010).

[36] L. Zhou, H. Song, K. Liu, Z. Luan, P. Wang, L. Sun, S. Jiang, H. Xiang, Y. Chen, J. Du, H. Ding, K. Xia, J. Xiao, and D. Wu, Observation of Spin-Orbit Magnetoresistance in Metallic Thin Films on Magnetic Insulators, Sci. Adv. 4, eaao3318 (2018).

[37] E. Saitoh, M. Ueda, H. Miyajima, and G. Tatara, Conversion of Spin Current into Charge Current at Room Temperature: Inverse Spin-Hall Effect, Appl. Phys. Lett. 88, 182509 (2006).

[38] K.-R. Jeon, C. Ciccarelli, H. Kurebayashi, J. Wunderlich, L. F. Cohen, S. Komori, J. W. A. Robinson, and M. G. Blamire, Spin-Pumping-Induced Inverse Spin-Hall Effect in $\mathrm{Nb} / \mathrm{Ni}_{80} \mathrm{Fe}_{20}$ Bilayers and Its Strong Decay Across the Superconducting Transition Temperature, Phys. Rev. Applied 10, 014029 (2018).

[39] A. Brataas, A. D. Kent, and H. Ohno, Current-Induced Torques in Magnetic Materials, Nat. Mater. 11, 372 (2012).

[40] V. Vlaminck and M. Bailleul, Current-Induced Spin-Wave Doppler Shift, Science 322, 410 (2008).

[41] V. Vlaminck and M. Bailleul, Spin-Wave Transduction at the Submicrometer Scale: Experiment and Modeling, Phys. Rev. B 81, 014425 (2010).

[42] O. V. Dobrovolskiy, R. Sachser, T. Brächer, T. Böttcher, V. V. Kruglyak, R. V. Vovk, V. A. Shklovskij, M. Huth, B. Hillebrands, and A. V. Chumak, Magnon-Fluxon
Interaction in a Ferromagnet/Superconductor Heterostructure, Nat. Phys. 15, 477 (2019).

[43] A. V. Chumak, V. I. Vasyuchka, A. A. Serga, and B. Hillebrands, Magnon Spintronics, Nat. Phys. 11, 453 (2015).

[44] M. A. Silaev, I. V. Bobkova, and A. M. Bobkov, Odd Triplet Superconductivity Induced by the Moving Condensate, arXiv:2001.02507.

[45] A. Manchon, H. C. Koo, J. Nitta, S. M. Frolov, and R. A. Duine, New Perspectives for Rashba Spin-Orbit Coupling, Nat. Mater. 14, 871 (2015).

[46] J. R. Raphael, R. Bindel, M. Pezzotta, J. Ulrich, M. Liebmann, E. Y. Sherman, and M. Morgenstern, Probing Variations of the Rashba Spin-Orbit Coupling at the Nanometre Scale, Nat. Phys. 12, 920 (2016).

[47] https://doi.org/10.17863/CAM.52983.

[48] K.-R. Jeon, C. Ciccarelli, H. Kurebayashi, L. F. Cohen, S. Komori, J. W. A. Robinson, and M. G. Blamire, Abrikosov Vortex Nucleation and Its Detrimental Effect on Superconducting Spin Pumping in $\mathrm{Pt} / \mathrm{Nb} / \mathrm{Ni}_{80} \mathrm{Fe}_{20} / \mathrm{Nb} / \mathrm{Pt}$ Proximity Structures, Phys. Rev. B 99, 144503 (2019).

Correction: The omission of the name of the 11th author has been fixed. The first displayed equation and the second inline equation afterward in Sec. III of the Appendix contained minor errors and have been set right. 